\title{
Propagating Waves Can Explain Irregular Neural Dynamics
}

\author{
Adam Keane ${ }^{1}$ and Pulin Gong ${ }^{1,2}$ \\ ${ }^{1}$ School of Physics and ${ }^{2}$ ARC Centre of Excellence for Integrative Brain Function, University of Sydney, NSW 2006, Australia
}

Cortical neurons in vivo fire quite irregularly. Previous studies about the origin of such irregular neural dynamics have given rise to two major models: a balanced excitation and inhibition model, and a model of highly synchronized synaptic inputs. To elucidate the network mechanisms underlying synchronized synaptic inputs and account for irregular neural dynamics, we investigate a spatially extended, conductance-based spiking neural network model. We show that propagating wave patterns with complex dynamics emerge from the network model. These waves sweep past neurons, to which they provide highly synchronized synaptic inputs. On the other hand, these patterns only emerge from the network with balanced excitation and inhibition; our model therefore reconciles the two major models of irregular neural dynamics. We further demonstrate that the collective dynamics of propagating wave patterns provides a mechanistic explanation for a range of irregular neural dynamics, including the variability of spike timing, slow firing rate fluctuations, and correlated membrane potential fluctuations. In addition, in our model, the distributions of synaptic conductance and membrane potential are non-Gaussian, consistent with recent experimental data obtained using whole-cell recordings. Our work therefore relates the propagating waves that have been widely observed in the brain to irregular neural dynamics. These results demonstrate that neural firing activity, although appearing highly disordered at the single-neuron level, can form dynamical coherent structures, such as propagating waves at the population level.

Key words: balanced excitation and inhibition; cerebral cortex; computer simulation; cross-correlation; propagating waves; synchrony

\section{Introduction}

Cortical neurons in vivo fire highly irregularly. Understanding the origin of this irregularity has been a long-standing topic of interest in neuroscience (Softky and Koch, 1993; Shadlen and Newsome, 1994; van Vreeswijk and Sompolinsky, 1996; Stevens and Zador, 1998). One prevalent view in accounting for this irregularity is that excitatory and inhibitory synaptic currents received by cortical neurons are approximately balanced. In this balanced condition, membrane potential follows a random walk and crosses the spiking threshold at random times (Shadlen and Newsome, 1994, 1998). Importantly, it has been shown that the balanced condition emerges naturally in randomly coupled neural networks and that, in such balanced networks, different neurons asynchronously emit spikes (van Vreeswijk and Sompolinsky, 1996). Along with variability in their spike timing, cortical neurons show firing rate fluctuations (Churchland et al., 2010), which can be captured by a balanced network model with clustered connections (Litwin-Kumar and Doiron, 2012).

Another view proposed to account for the variability of cortical firing activity is that presynaptic population activity is highly synchronized, and such synchronized activity causes large fluctuations in synaptic inputs and the resultant variability of neural

Received April 22, 2014; revised Nov. 17, 2014; accepted Nov. 26, 2014.

Author contributions: A.K. and P.G. designed research; A.K. and P.G. performed research; A.K. and P.G. analyzed data; A.K. and P.G. wrote the paper.

We thank Prof. Paul Martin for helpful discussion and comments on the manuscript.

The authors declare no competing financial interests.

Correspondence should be addressed to Dr. Pulin Gong, School of Physics, University of Sydney, NSW 2006, Australia. E-mail: pulin.gong@sydney.edu.au.

DOI:10.1523/JNEUROSCI.1669-14.2015

Copyright $\odot 2015$ the authors $\quad 0270-6474 / 15 / 351591-15 \$ 15.00 / 0$ spike timing (Stevens and Zador, 1998). In this proposal, synchronized synaptic inputs cause membrane potential to exhibit large, occasional excursions in amplitude. Membrane potential therefore has a non-Gaussian distribution instead of the Gaussian one found in random walk models (Shadlen and Newsome, 1998). Such synchronized synaptic inputs and non-Gaussian membrane potential dynamics have been widely observed in cortical neurons (DeWeese and Zador, 2006; Tan et al., 2014). However, the network mechanisms that give rise to these dynamics remain unknown. Furthermore, whether and how the two leading views (i.e., the view of balanced excitation and inhibition and that of synchronized inputs) can be reconciled to gain a comprehensive understanding of cortical dynamics remain unresolved.

Here, we propose an alternative view on the basis of propagating wave patterns emerging from $2 \mathrm{D}$, spatially extended spiking neural networks. As these wave patterns sweep past neurons, they generate highly synchronized synaptic inputs. Propagating wave patterns with complex dynamics only emerge when excitation and inhibition are balanced. Our model therefore reconciles the two leading views regarding the variability of neural dynamics. In addition, our model is able to account for a range of experimental observations, including the variability of spike timing, slow fluctuations of firing rates (Churchland et al., 2010), synchronized synaptic inputs with inhibition lagging behind excitation by several milliseconds (Okun and Lampl, 2008), and membrane potentials with non-Gaussian dynamics (DeWeese and Zador, 2006) and correlated fluctuations (Gentet et al., 2010).

Evidence of the presence of propagating waves in the brain has been rapidly accumulating (Rubino et al., 2006; Ferezou et al., 2007; Wu et al., 2008; Sato et al., 2012). Our work relates these widely observed population activity patterns to variable neuronal 
dynamics, indicating that structured activity patterns, such as propagating waves, at the level of neural circuits can emerge from the highly variable firing activity of individual neurons.

\section{Materials and Methods}

A spatially extended, conductance-based spiking neural circuit. We consider a 2D network of $N \times N$ coupled, conductance-based integrate-andfire neurons consisting of $80 \%$ excitatory and $20 \%$ inhibitory neurons. Both excitatory and inhibitory neurons are evenly spaced, and the spacing between inhibitory neurons is twice the spacing between excitatory neurons. We denote the voltage of a neuron at integer coordinates $(i, j)$ at time $t$ as $V_{i j}(t)$, with dynamics given by the following:

$$
C \frac{d}{d t} V_{i j}(t)=-g_{L}\left[V_{i j}(t)-V_{L}\right]-g_{i j}^{E}(t)\left[V_{i j}(t)-V_{E}\right]-g_{i j}^{I}(t)\left[V_{i j}(t)-V_{I}\right],
$$

where the capacitance is $C=1 \mu \mathrm{F}$, the leak conductance is $g_{L}=50 \mu \mathrm{S}$, and the reversal potentials are $V_{L}=-70 \mathrm{mV}, V_{E}=0 \mathrm{mV}$, and $V_{I}=-80$ $\mathrm{mV}$ for the leak, excitatory, and inhibitory conductance, respectively (Koch, 1999). If the voltage of a neuron reaches the threshold of $V_{T}=$ $-55 \mathrm{mV}$, a spike is generated and the voltage is reset to the reset potential $V_{R}=-70 \mathrm{mV}$ for a refractory period $\tau_{\text {ref }}=5 \mathrm{~ms}$. The synaptic conductances are as follows:

$$
g_{i j}^{\lambda}(t)=F^{\lambda}+\sum_{i^{\prime} j^{\prime}} K_{i j, i^{\prime} j^{\prime}}^{\lambda^{\prime}} \sum_{l} G^{\lambda^{\prime}}\left(t-T_{i^{\prime} j^{\prime}}^{l}\right),
$$

where $\lambda, \lambda^{\prime}=E, I$ are the excitatory and inhibitory conductances, respectively, and $T_{i^{\prime} j^{\prime}}^{l}$ is the time of the $l$-th spike emitted by the afferent neuron located at $\left(i^{\prime}, j^{\prime}\right)$. The constant external inputs are $F^{E}=15 \mu \mathrm{S}$ and $F^{I}=2$ $\mu \mathrm{S}$, but as shown in Results, our model is not sensitive to these values. The time course of the postsynaptic conductance is given by the following:

$$
G^{\lambda}(t)=\frac{\exp \left(-t / \tau_{d}^{\lambda}\right)-\exp \left(-t / \tau_{r}^{\lambda}\right)}{\tau_{d}^{\lambda}-\tau_{r}^{\lambda}},
$$

with rise times $\tau_{r}^{E}=0.5 \mathrm{~ms}$ and $\tau_{r}^{I}=0.5 \mathrm{~ms}$, and decay times $\tau_{d}^{E}=2.0 \mathrm{~ms}$ and $\tau_{d}^{I}=7.0 \mathrm{~ms}$. The denominator is a normalization factor such that $\int_{0}^{\infty} G^{\lambda}(t) d t=1$. The coupling strength between any two neurons located at $(i, j)$ and $\left(i^{\prime}, j^{\prime}\right)$ is given by the following:

$$
K_{i j, i^{\prime} j^{\prime}}^{\lambda}= \begin{cases}W_{E} \exp \left(-d_{i j, i^{\prime} j^{\prime} j^{\prime}}^{2} / \sigma_{E}\right) & \text { if } \lambda=E, \\ W_{I} & \text { if } \lambda=I,\end{cases}
$$

where $d_{i j, i^{\prime} j^{\prime}}$ is Euclidean distance between neurons on a square lattice with periodic boundary conditions. Because the interactions between neurons in real neural systems have finite ranges, connections in the model are constrained to $\left|d_{i, i^{\prime} j^{\prime}}\right| \leq D^{\lambda}$, where $D^{E}=10$ for excitatory neurons and $D^{I}=15$ for inhibitory neurons. Empirical evidence has been accumulating to show that neural connectivity decays with distance; for instance, in Markov et al. (2011), it was found that the coupling strength is an exponential function of distance; and in Levy and Reyes (2012), it was reported that the connection probability of neurons is a Gaussian function of distance. As a first approximation to such distancedependent coupling rules, excitatory neurons in our model are coupled to all other neurons within the coupling range with a strength following a Gaussian profile (Eq. 4). Because anatomic evidence suggests that inhibitory connections to pyramidal neurons are nonspecific (Fino and Yuste, 2011), in Equation 4 we use a homogeneous inhibitory coupling strength $W_{I}$ with all inhibitory neurons connected to each other within the coupling range. We use excitatory coupling value $W_{E}=0.23$ with a spatial scale of $\sigma_{E}=12$, unless stated otherwise; however, as illustrated below, our results are robust to parameter variations. The inhibitory coupling $W_{I}$ is a free parameter that controls the balance of excitation and inhibition. Each neuron receives the same number of connections (i.e., 316 excitatory connections and 180 inhibitory connections, approximately one-tenth of the average number of connections found in the cortex) (Braitenberg and Schüz, 1998).
We use a network size of $300 \times 300$ but have found that the results are not sensitive to the size of the network. The network model is simulated by using the Euler method with a time step $d t=0.05 \mathrm{~ms}$ (Press et al., 2007). The initial membrane potentials are randomly chosen from values between the resting potential $\left(V_{R}=-70 \mathrm{mV}\right)$ and the threshold potential $\left(V_{T}=-55 \mathrm{mV}\right)$. Each trial is run for $7.5 \mathrm{~s}$ with the first $1.5 \mathrm{~s}$ excluded as transient time.

To compare our results with previous balanced networks with random coupling topology, each connection is randomly rewired to another neuron in the network. The reconnection is independent of distance between neurons, but self-connection and multiple connections from the same neuron are excluded. For this randomly rewired network, individual neurons receive a variable number of connections with the average number the same as the regular network. To investigate the effects of partial rewiring, each afferent excitatory connection is rewired with a probability $P$; for the randomly coupled network $P=100 \%$.

To demonstrate the role of intrinsic network dynamics in generating variable neural activity, we mostly focus on a model with deterministic external input (i.e., $F^{\lambda}$ in Eq. 2 is a constant). However, because noise is ubiquitous in the brain (Faisal et al., 2008), we also consider noisy inputs to individual neurons to demonstrate that propagating wave patterns can still emerge from our model. For this case, if a neuron is not in the refractory period, at each time step it fires with a certain probability, resulting in random spontaneous firing activity with a rate of $2 \mathrm{~Hz}$, as found in real cortical neurons (Koch and Fuster, 1989). Noisy inputs are also used for the randomly connected network.

Data analysis. Statistics of spike trains, firing rates, synaptic conductances, and membrane potentials of each neuron are calculated to compare our modeling study with experiments. To that end, we measure the coefficient of variation (CV) of interspike intervals, the Fano factor of spike counts, and the cross-correlation and excess kurtosis of synaptic conductance and membrane potential. All results are calculated by using 2400 randomly chosen excitatory neurons in each of 12 trials, unless otherwise specified.

Let $0<t_{i j}^{1}<t_{i j}^{2}<\ldots<t_{i j}^{L}<T$ denote a train of $L$ spikes that are emitted by the neuron at position $(i, j)$ during a trial of duration $T$; then $X_{i j}^{1}, X_{i j}^{2}, \ldots, X_{i j}^{L-1}$ is the sequence of the $L-1$ observed interspike intervals (ISIs) $X_{i j}^{l}=t_{i j}^{l+1}-t_{i j}^{l}$ where $l$ indicates the $l$-th spike. Irregular spiking is expressed by a variable length of the ISIs. To quantify this variability, we calculate the CV of the ISIs of the neuron at $(i, j)$ :

$$
\mathrm{CV}_{i j}=\frac{\sqrt{\operatorname{var}\left(X_{i j}^{l}\right)}}{\left\langle X_{i j}^{l}>\right.},
$$

where $\left\langle X_{i j}^{l}\right\rangle$ and $\operatorname{var}\left(X_{i j}^{l}\right)$ are the mean and variance of the ISIs, respectively. A periodic process, such as the ticking of a clock, has a CV of 0 ; a Poisson process, in which the timing of one event is independent of any other, has a CV of 1. Multiple observations of neurons at different positions results in an ensemble of spike trains, each with its own CV. The CVs can be averaged to find the overall variability of ISIs.

The CV of ISIs signifies intratrial variability on a relatively short time scale on the order of the length of a typical ISI. By contrast, the trial-totrial variability is measured by the Fano factor of the spike count $N_{i j}(t)$, which is the number of spikes emitted by a neuron within a fixed time interval from $t$ to $t+\Delta t$. The Fano factor of a neuron at $(i, j)$ is then:

$$
\mathrm{FF}_{i j}(t)=\frac{\operatorname{var}\left(N_{i j}(t)\right)}{<N_{i j}(t)>}
$$

where $\left\langle N_{i j}(t)\right\rangle$ and $\operatorname{var}\left(N_{i j}(t)\right)$ are the mean and variance of the spike count across repeated trials with random initial conditions. The Fano factor of a homogeneous (with a constant rate of events) Poisson process is 1 .

To measure the correlations between pairs of spiking neurons, we calculate the cross-correlation of their spike count. The normalized cross-correlation (cross-covariance) of the spike counts, $N_{i j}(t)$ and $N_{i^{\prime} j^{\prime}}(t)$, of two neurons at $(i, j)$ and $\left(i^{\prime}, j^{\prime}\right)$ is calculated according to the following: 


$$
\mathrm{CC}_{i j, i^{\prime}, j^{\prime}}(\tau)=\frac{\sum_{l=1}^{L}\left(N_{i j}\left(t^{l}\right)-<N_{i j}\left(t^{l}\right)>\right)\left(N_{i^{\prime} j^{\prime}}\left(t^{l}+\tau\right)-<N_{i^{\prime} j^{\prime}}\left(t^{l}\right)>\right)}{\sqrt{\operatorname{var}\left(N_{i j}\left(t^{l}\right)\right) \operatorname{var}\left(N_{i^{\prime} j^{\prime}}\left(t^{l}\right)\right)}},
$$

where $\left\langle N_{i j}\left(t^{t}\right)\right\rangle$ and $\left\langle N_{i^{\prime} j^{\prime}}\left(t^{l}\right)\right\rangle$ are the means of the spike counts, $\operatorname{var}\left(N_{i j}\left(t^{l}\right)\right)$ and $\operatorname{var}\left(N_{i^{\prime} j^{\prime}}\left(t^{l}\right)\right)$ are the variances, $L$ is the total number of samples in the time series, and $t^{l}=(l-1) \delta t$ with $\delta t=1 \mathrm{~ms}$. The number of spikes are calculated over $\Delta t=50 \mathrm{~ms}$ time windows. For $i=i^{\prime}, j=j^{\prime}$ (i.e., the auto-correlation), the two identical signals would have a value of $C C_{i j, i^{\prime} j^{\prime}}(\tau=0)=1$ at zero time lag. Nonidentical traces result in values of $C C_{i j, i^{\prime} j^{\prime}}(\tau=0)$ between -1 and 1 , which indicates negative and positive correlations, respectively. At zero time lag, the measured value is identical to the Pearson product-moment correlation coefficient, which is a measure of the instantaneous correlation between two variables. The cross-correlation can be calculated for the membrane potential $\left(V_{i j}(t)\right.$ and $\left.V_{i^{\prime} j^{\prime}}(t)\right)$ and synaptic conductance $\left(g_{i j}^{E}(t)\right.$ and $\left.g_{i^{\prime} j^{\prime}}^{E}(t)\right)$ of pairs of neurons in the same way as described above.

To characterize the shape of the distribution of membrane potentials, we compute their excess kurtosis. For a neuron at position $(i, j)$, this is given by the following:

$$
\operatorname{kurtosis}\left(V_{i j}\right)=\frac{L \sum_{l=1}^{L}\left(V_{i j}\left(t^{l}\right)-<V_{i j}\left(t^{l}\right)>\right)^{4}}{\left(\sum_{l=1}^{L}\left(V_{i j}\left(t^{l}\right)-<V_{i j}\left(t^{l}\right)>\right)^{2}\right)^{2}}-3,
$$

where $\left\langle V_{i j}\left(t^{l}\right)\right\rangle$ is the mean value of voltage, $L$ is the total number of samples in the time series, and $t^{l}=(l-1) \delta t$ with $\delta t=1 \mathrm{~ms}$. The excess kurtosis (which we shall henceforth refer to as the kurtosis) is a measure of the shape of a distribution compared with a Gaussian; it is $>0$ for a distribution with more outliers (i.e., it is heavy-tailed) or values close to the mean, and $<0$ for distributions with more values between the mean and the outliers (i.e., it has heavy shoulders); for a Gaussian distribution, it is 0 (DeCarlo, 1997; DeWeese and Zador, 2006). Large, occasional deviations from the resting membrane potential lead to a heavy-tail distribution, and we use the kurtosis to quantify this, excluding the reset values of membrane potential that occur in the refractory period. The kurtosis of the synaptic conductance $g_{i j}^{E}(t)$ of each neuron is calculated in the same manner.

The spatiotemporal activity of our spatially extended network exhibits propagating wave patterns with complex dynamics, as described in Results. One of the salient features of these wave patterns is that they are localized, meaning that neurons that are spiking in a certain interval are adjacent, and individual patterns are clearly separated from each other. Based on this property, we devise an automatic method to identify these patterns. We first choose a time window of duration $5 \mathrm{~ms}$ to detect enough spikes that are adjacent to each other, and we then use a flood-fill algorithm to classify groups of adjacent neurons as one pattern (Burger and Burge, 2008).

There are two kinds of propagating wave pattern emerging from our network model, which are described in Results: patchy and crescentshaped waves. A significant difference between them is that the center of the former contains random firing activity, which results in gaps (i.e., holes) between firing neurons, whereas the latter does not. This property can be quantified by the Euler characteristic, which is the difference between the number of connected regions and the number of their holes (Burger and Burge, 2008); thus, for the crescent-shaped waves, the Euler characteristic is 1; and for the patchy patterns, it is $<1$. The Euler characteristic is calculated by the "bweuler" routine in MATLAB (MathWorks) (Pratt, 1991) and can be used to reliably distinguish the two types of propagating wave patterns.

After identifying these propagating wave patterns automatically, we characterize their collective dynamics by calculating the center-of-mass position $\left(I_{M}(t), J_{M}(t)\right)$ of each pattern:

$$
I_{M}(t)=\frac{1}{M_{f}} \sum_{L=1}^{M_{f}} i_{M}^{L}(t), \quad J_{M}(t)=\frac{1}{M_{f}} \sum_{L=1}^{M_{f}} j_{M}^{L}(t)
$$

where $i_{M}^{L}(t)$ and $j_{M}^{L}(t)$ are the $i$ and $j$ positions of the $L$ th neuron that is firing at time $t$ in the $M$ th pattern, and $M_{f}$ is the total number of firing neurons within this pattern.

We then use the mean-squared displacement (MSD) to quantify the motion of each pattern:

$$
\operatorname{MSD}(\tau)=<\left(I_{M}(t+\tau)-I_{M}(t)\right)^{2}+\left(J_{M}(t+\tau)-J_{M}(t)\right)^{2}>
$$

where $\tau$ is the time increment, and $<>$ represents averaging over time. If a pattern travels ballistically, that is, it moves in the same direction at each time step, $\operatorname{MSD}(\tau) \propto t^{\alpha}$ where $\alpha=2$. If the motion of a pattern is normally diffusive (Brownian) (i.e., it is equally likely to move in any direction at each time step), then $\alpha=1$.

\section{Results}

\section{Dynamic wave patterns emerging from balanced, spatially extended networks}

The balance of a neuron often refers to the approximately equivalent amounts of inhibitory and excitatory synaptic inputs that it receives (van Vreeswijk and Sompolinsky, 1996, 1998; Salinas and Sejnowski, 2000; Xue et al., 2014). This can be measured by the ratio $\beta=\left\langle\overline{c_{i j}^{I}}\right\rangle /\left\langle\overline{c_{i j}}\right\rangle$ where excitatory current $c_{i j}^{E}(t)=g_{i j}^{E}(t)\left|V_{i j}(t)-V_{E}\right|$, inhibitory current $c_{i j}^{I}(t)=g_{i j}^{I}(t)\left|V_{i j}(t)-V_{I}\right|, \quad<>$ indicates averaging over time excluding refractory periods, and the overbar represents averaging across neurons. When $\beta \approx 1$ (i.e., excitatory and inhibitory inputs are approximately equilibrated), we refer to this as the balanced condition. When $\beta \approx 1$, the randomly coupled network (see Materials and Methods) generates asynchronous dynamics without any structured patterns, as found in previous balanced networks (van Vreeswijk and Sompolinsky, 1996; Renart et al., 2010). In this balanced state, individual neurons fire irregularly (Fig. 1a), and the distribution of membrane potential is Gaussian.

In contrast, the balanced, spatially extended network generates spatiotemporal patterns with complex dynamics. As shown in Figure $1 b, c, d$, these patterns include several localized, crescent-shaped waves that move rapidly, and localized, patchy patterns that slowly wander around (outlined by the green boxes). The initiation sites and subsequent trajectories of both types of pattern are seemingly random, and the patterns interact when they approach each other; these interactions include repulsive collisions, and partial and full annihilations. Because both types of pattern can propagate over the network, we generally refer to them as propagating wave patterns. Notably, propagating wave patterns with random initiation sites and propagation directions have been observed in the spontaneous activity of visual cortex of rats (Han et al., 2008, their Fig. 2b) and in the sensorimotor cortex of behaving mice (Ferezou et al., 2007). Multiple wave patterns that sweep across the cortex have been found in the visual cortex of cats (Arieli et al., 1995, 1996), and the coexistence of waves and localized patchy patterns has been observed in the barrel cortex of rodents (Petersen et al., 2003).

When the network input is unbalanced $(\beta \leq 0.95)$ and disinhibited, wave patterns with more regular, global properties emerge. In particular, global ring or target waves occur periodically, with frequency proportional to the external input. Alternatively, depending on the initial conditions, the network sometimes develops plane waves that propagate in a preferred direction, or spiral waves, the centers of which can spread across the entire lattice (Fig. 1e). This is largely consistent with recordings in cortical slices and intact neocortex of rats: when neurons were disinhibited using bicuculline to block GABA receptors, 


\section{a}

Random connectivity

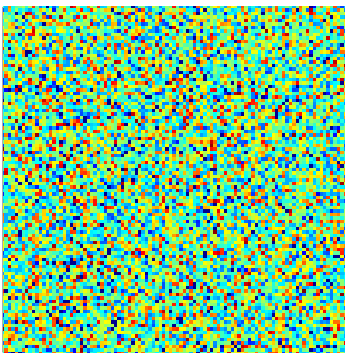

d Regular connectivity, $1700 \mathrm{~ms}$

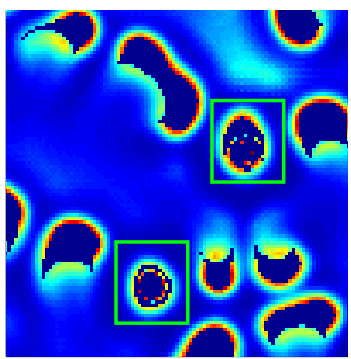

g Excitation > inhibition, $3020 \mathrm{~ms}$

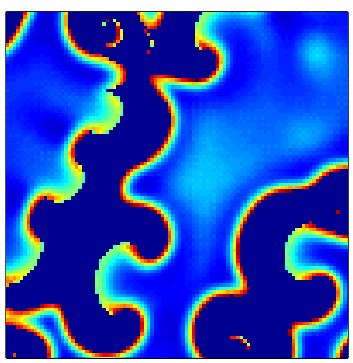

j Inhibition > excitation, $4500 \mathrm{~ms}$

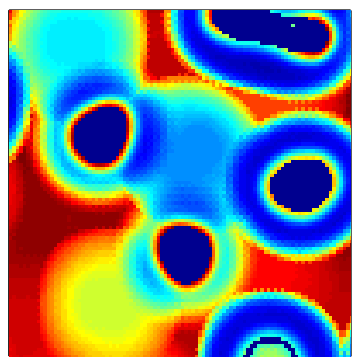

b

Regular connectivity, $1500 \mathrm{~ms}$

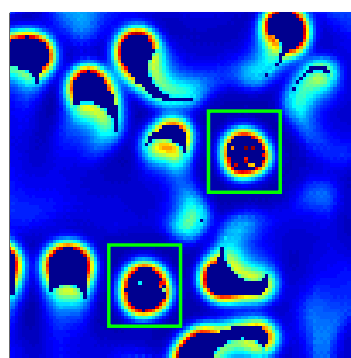

e

Regular connectivity, excitation dominates

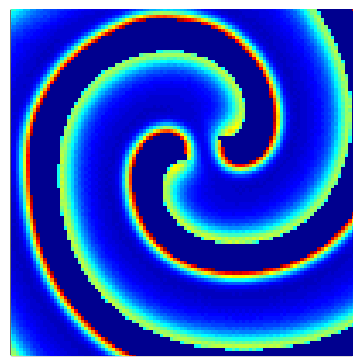

h

Excitation > inhibition, $4500 \mathrm{~ms}$

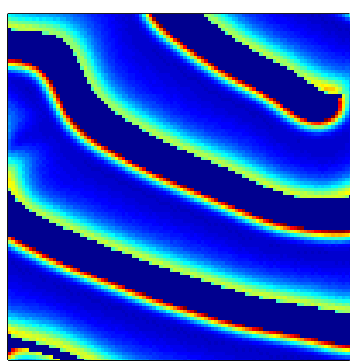

k

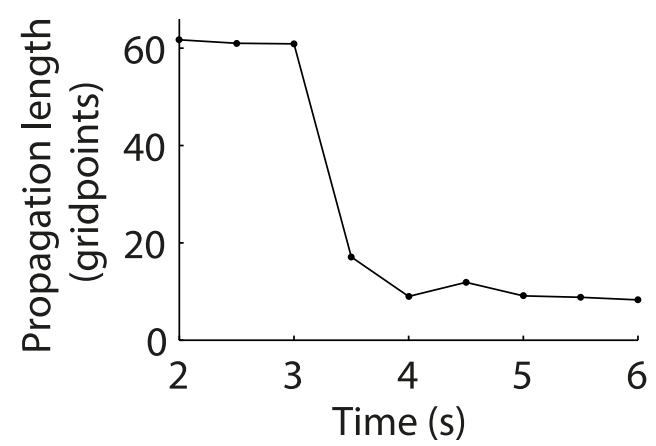

Regular connectivity, $1600 \mathrm{~ms}$

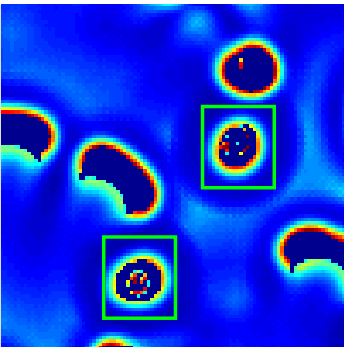

f Excitation > inhibition, $3010 \mathrm{~ms}$
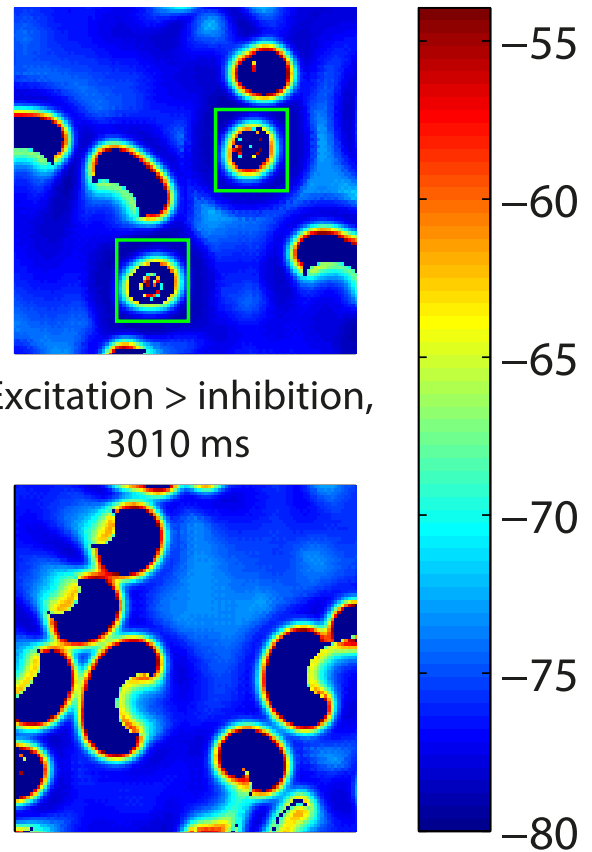

Excitation > inhibition

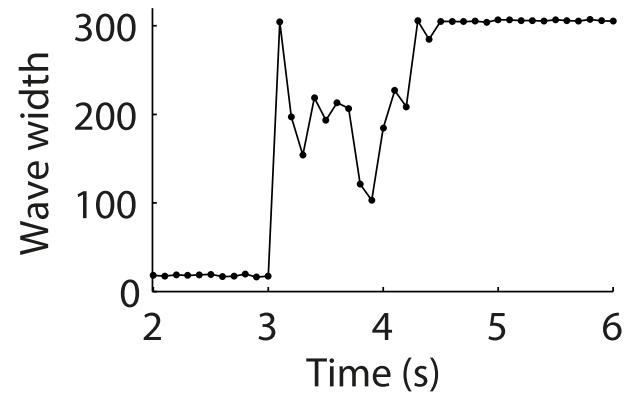

I Regular connectivity, noisy input

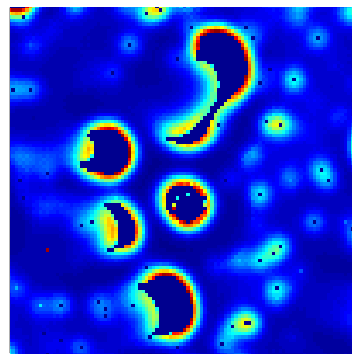

Figure 1. The formation of propagating wave patterns with complex dynamics depends upon a balance of excitation and inhibition, and upon spatially extended coupling. Colors in the images represent membrane potential between inhibitory reversal $V_{I}=-80 \mathrm{mV}$ (blue) and threshold $V_{T}=-55 \mathrm{mV}$ (red), as indicated by the color bar. Each image is of a network of $300 \times 300 \mathrm{neurons}$, but only a $100 \times 100$ region is shown for clarity. $\boldsymbol{a}$, Snapshot of spatiotemporal activity in the randomly connected, balanced network. For this case, there are no structured patterns. $\boldsymbol{b}$ - $\boldsymbol{d}$, Snapshots of spatiotemporal patterns in the balanced, spatially extended network. Patterns take the form of multiple, localized, crescent-shaped propagating waves and wandering patches, which are outlined by the green boxes. The patterns may interact in a variety of ways, such as mutual or partial annihilations, in which one or both patterns disappear; deflection, in which patterns move in opposite directions; and fission, in which a wave splits into two.e, A spiral wave formed in the unbalanced network in which excitation dominates; the center of the spiral wave drifts across the network. $\boldsymbol{f}$ - $\boldsymbol{h}$, Snapshot of spatiotemporal activity when the balanced condition in a network is broken so that excitation dominates. The separation between the waves decreases, and then nearby waves merge; this effect persists until plane waves form. $\boldsymbol{i}$, Average width of the waves as a function of time. The balanced condition is broken at $3 \mathrm{~s}$, and for this case the excitation is dominant. $\boldsymbol{j}$, Spatiotemporal activity when balance in a network is broken so that inhibition dominates; patterns tend to dissipate quickly such that they cannot propagate over long distances. $\boldsymbol{k}$, Propagation distances of the wave patterns as a function of time. The balanced condition is broken at $3 \mathrm{~s}$, and inhibition dominates in this case. I, Snapshot of the spatiotemporal activity of the spatially extended network when noisy inputs are included. Despite the presence of random inputs, propagating wave patterns are still able to form from the network. 
alternative patterns of ring, plane, and spiral waves were observed (Huang et al., 2004, 2010).

To further illustrate that balanced excitation and inhibition are needed for the formation of propagating wave patterns with complex dynamics, we break the balanced condition in the network to study the changes in these patterns. For this purpose, the model is run for the usual transient time and then after $3 \mathrm{~s}$, the inhibitory connection strength $W_{I}$ is altered to obtain either an excitation or an inhibition dominated case. For the excitation dominated case, the typical changes of these patterns after breaking the balance are shown in Figure $1 f, g$, $h$ : the separation between these localized wave patterns decreases, and then nearby waves merge with each other; this process continues until eventually global plane waves are formed (Fig. $1 h$ ). To quantify such changes, we calculate the average width of the wave patterns identified by our algorithm (see Materials and Methods) as a function of time. Figure $1 i$ shows that the size of the waves increases rapidly after breaking the balanced condition and continues to increase until all the patterns are merged into a plane wave. On the other hand, after breaking the balanced condition to obtain an inhibition dominated case, the wave patterns change from ones with relatively long range propagation to ones tending to disappear shortly after they are formed (i.e., they are short-lived due to the strong inhibition) (Fig. 1j). Based on this observation, we calculate the average propagation length (500 ms time windows) of each pattern as a function of time. Figure $1 k$ shows that, as expected, the propagation length of the waves rapidly decreases after the balance is broken until it reaches a certain value that is quite small relative to the size of the network.

In our network model, the balanced state and the resultant propagating wave patterns emerge without fine tuning of parameters. For instance, changing the values of $F^{E}$ and $F^{I}$ still leads to the balanced state as long as $F^{E}$ is high enough relative to $F^{I}$ such that individual neurons can fire even without recurrent synaptic inputs. The population-averaged firing rates increase nearly linearly as a function of external input strength $\left(F^{E}\right)$, as has been found for randomly connected, balanced networks (van Vreeswijk and Sompolinsky, 1996). Additionally, when the neurons in our network receive stochastic inputs (see Materials and Methods), they still settle to a balanced state and the network is able to form propagating wave patterns (Fig. $1 l$ ), as found in the network with a constant external input. Moreover, the strengths of recurrent excitation $\left(W_{E}\right)$ and inhibition $\left(W_{I}\right)$ have a relatively broad range over which the balanced condition is preserved; for instance, given $W_{E}=0.23$, the network is balanced for $0.23 \leq W_{I} \leq 0.35$. In a recent study of neural field models (Rosenbaum and Doiron, 2014), it was found that, in the continuum limit, balanced firing rate solutions require that the spatial spread of external inputs be broader than that of recurrent excitation, which in turn should be greater than or equal to that of recurrent inhibition; however, this condition is not satisfied for finite-size networks, and crucially, propagating wave activity cannot be captured by the mean field models, as pointed out by Rosenbaum and Doiron (2014). In our study of the finite-size spiking neural network with emergent propagating wave patterns, the balanced condition with the ratio of average excitatory current and inhibitory current close to 1.0 is quite robust, and these wave patterns underlie variable firing activity of neurons as shown in the following section.

\section{Spikes of neurons exhibit a doubly stochastic process}

We next characterize the dynamics of the propagating wave patterns and show that these dynamics cause the doubly stochastic process of neural firing activity, with variability in spike timing and slow fluctuations in firing rates (Churchland et al., 2010, 2011). First, the center of mass of each pattern is calculated and tracked over time (see Materials and Methods). Figure $2 a, b$ shows the typical trajectories of crescent-shaped waves and patchy patterns, respectively; both patterns appear to have random initiation sites and trajectories. They have distinct dynamics, with crescent-shaped waves propagating over long distances and patchy patterns wandering around a particular area. By calculating the displacement of centers of mass for each pattern, we find that the crescent-shaped waves have a mean speed of $2.0 \pm$ 0.6 gridpoints/ms (Fig. $2 c$ ). We also change the connectivity density (i.e., the number of connections each neuron receives over a given distance) and find that the average speed of the waves increases monotonically as the density increases.

To further quantify the dynamics of these patterns, we calculate their MSD (see Materials and Methods). For the crescentshaped waves, MSD appears to be linear on a log-log scale (Fig. $2 d$, red line), which implies that it is a power function of time increment $\tau$ such that $M S D(\tau) \propto \tau^{\alpha}$. By using nonlinear leastsquares fitting via the Levenberg-Marquardt algorithm (Press et al., 2007), we obtain an exponent of $\alpha=1$.9. For Brownian motion, the MSD is a linear function of time $(\alpha=1)$; when $\alpha \neq 1$, a diffusion process has a nonlinear relationship to time and is called anomalous diffusion. In particular, if $1<\alpha<2$, it is a superdiffusive process; and if $0<\alpha<1$, it is a subdiffusive process (Metzler and Klafter, 2000). This result therefore indicates that the crescent-shaped waves move in a superdiffusive manner. Anomalous superdiffusion indicates that the motion of these waves is not fully random as is the case for Brownian motion and that, instead, there are long-range correlations in their propagations (Metzler and Klafter, 2000). For the patchy patterns that wander around, $\operatorname{MSD}(\tau) \propto \tau^{\alpha}$ and $\alpha=1.0$ (using nonlinear least-squares fitting) for $\tau>10 \mathrm{~ms}$, indicating that these patterns move in a normally diffusive manner over long time scales and that they move much slower than crescent-shaped waves (Fig. $2 d$, blue line).

Figure $3 a$ shows that neurons within the network exhibit dynamic transitions between low-activity and high-activity states. These transitions appear to coexist with variable spike timing of individual neurons, which is a characteristic feature of a doubly stochastic process (Churchland et al., 2011; Churchland and Abbott, 2012; Litwin-Kumar and Doiron, 2012). To quantify the variability of spike timing, we first calculate the CV of ISIs as outlined in Materials and Methods. The average value of CVs across all trials is $1.1 \pm 0.1$, which is in agreement with experimental studies (Softky and Koch, 1993; Holt et al., 1996). However, a high CV may reflect fluctuations in firing rates as well as variability in the timing of individual spikes. To quantify such fluctuations in firing rates, we calculate the spike count Fano factor (see Materials and Methods) for each neuron by using a fixed time window of $\Delta t=100 \mathrm{~ms}$, which yields a mean value of $1.4 \pm 0.5$. Fano factors with values $>1.0$ have been observed in many cortical systems (Tolhurst et al., 1983; Britten et al., 1993; Churchland et al., 2011) and are evidence for the fluctuations of neural firing rates. The fluctuations of firing rates over long time scales are further demonstrated by calculating Fano factors as a function of time window. As shown in Figure $3 b$, Fano factor increases linearly as $\Delta t$ increases; this is because of the transitions between high and low-activity states that happen over long time scales (Churchland et al., 2011). A recent modeling study of balanced networks with clustered connections has found similar doubly stochastic firing activity and transitions between high and 

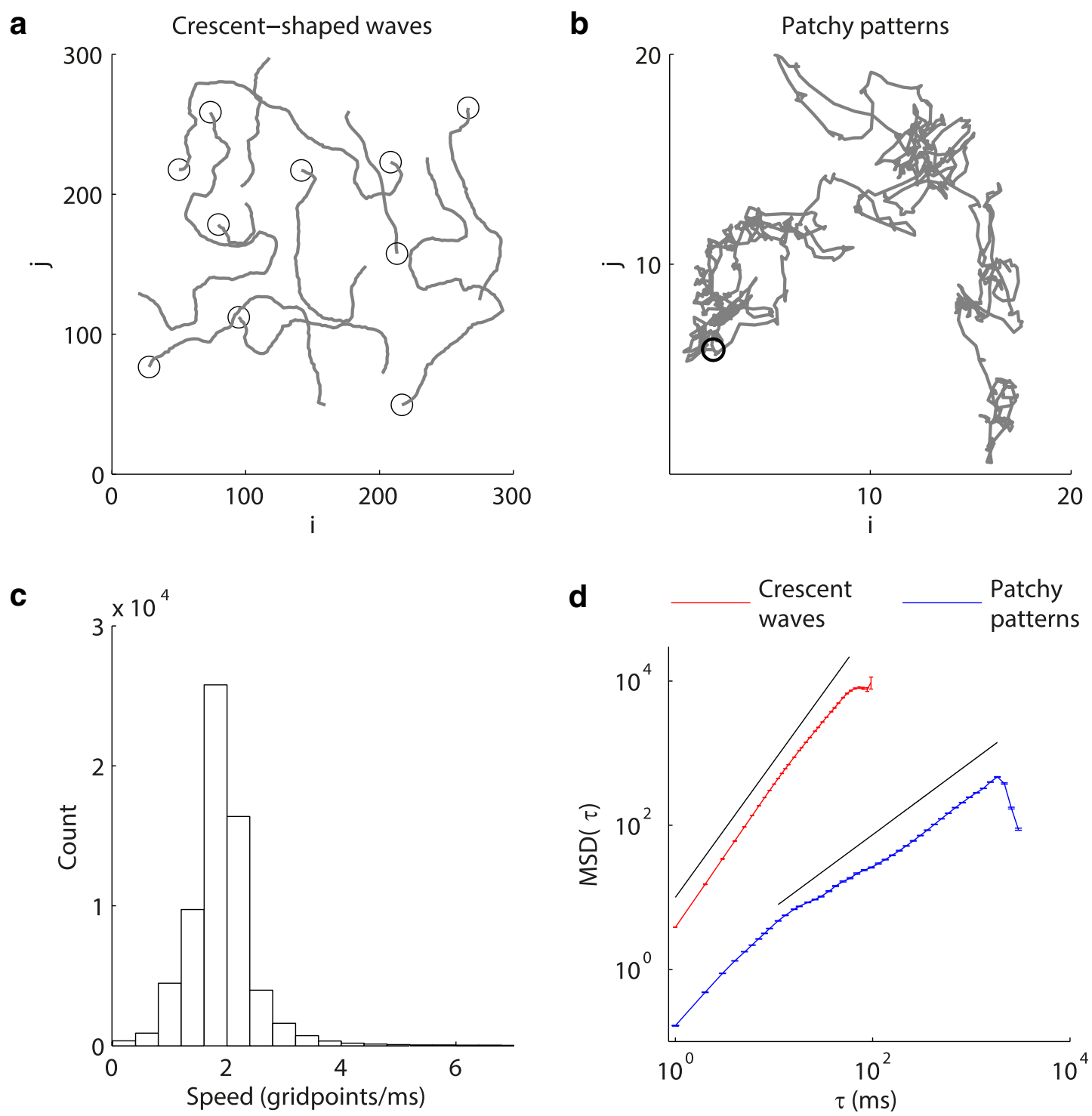

Figure 2. Dynamic properties of propagating wave patterns. $\boldsymbol{a}$, A sample of the trajectories of crescent-shaped waves. Both the initiation sites (black circles) and propagation paths (gray lines) of these waves appear to be random. The trajectories are terminated as a result of interactions with other waves or as a result of the wave fissioning. $\boldsymbol{b}$, Trajectory of a typical patchy pattern with initiation site depicted by the circle. The pattern tends to "wander" around a particular area and covers a smaller region than the crescent-shaped waves (note the smaller $20 \times 20$ scale of this figure). c, Distribution of the speeds of the crescent-shaped waves, which has a mean of $2.0 \mathrm{gridpoints} / \mathrm{ms}$. $\boldsymbol{d}$, Mean-squared displacement $M S D(\tau)$ as a function of time increment $\tau$. Red line indicates the MSD for crescent-shaped waves; a fit to the straight-line part in the log-log plot (indicated by the black line above) gives $M S D(\tau) \propto \tau^{\alpha}$ with $\alpha=1.9$ (nonlinear least-squares fitting), indicating the superdiffusive dynamics of crescent-shaped waves. To confirm that the crescent waves propagate in a superdiffusive way rather than a ballistic way $(\alpha=2)$, we compare our fit against a quadratic fit by calculating the Bayes factor of $B=P(D \mid M) / P\left(D \mid M_{0}\right)$ where $P(D \mid M)$ is the likelihood of the power law fit $M$ with $\alpha=1.9$ given the data $D$, and $P\left(D \mid M_{0}\right)$ is the likelihood of the quadratic fit $M_{0}$; we obtain $B>1000$, indicating that the superdiffusive case is much more likely than the ballistic case. Blue line indicates the MSD for patchy patterns: for $t>10$ $\mathrm{ms}$, the slope of the line of best fit (the black line above) is $\alpha=1.0$, which indicates Brownian dynamics over long time scales.

low firing activity states (Litwin-Kumar and Doiron, 2012), in which such transitions are caused by spontaneous switching dynamics between different attractors. However, for our case, the dynamics of the propagating wave patterns cause these transitions; this mechanism is illustrated below.

\section{Propagating wave patterns generate irregular spiking activity} We next consider the relationship between the spiking activity of individual neurons and the collective dynamics of propagating wave patterns. By tracking the motion of individual patterns, we observe that, when a crescent-shaped wave front moves toward a certain area, neurons in the area would receive synaptic inputs from neurons that are firing in the front, and their membrane potentials are depolarized such that they may subsequently fire.
Because the wave moves in a superdiffusive way, it leaves that location rapidly, which means that neurons around that location fire no more than once. To verify this, we consider a $5 \times 5$ subgrid with 25 neurons, which is smaller than the area of a typical wave pattern and thus cannot contain more than one wave trajectory at a time. We then calculate over all trials the number of neurons within this grid that would fire when a wave passes by. The resultant distribution, shown in Figure $3 c$, has a mean value of 11.2, meaning that, when a wave passes by, only a fraction of neurons in the grid fire. The complex dynamics of the waves, namely, their random origins and trajectories (Fig. $2 a$ ), results in the irregular passage of these wave fronts around that location; this causes the high variability of the spike times indicated by the CV. Because the waves tend to leave each location rapidly and cause only a 
a
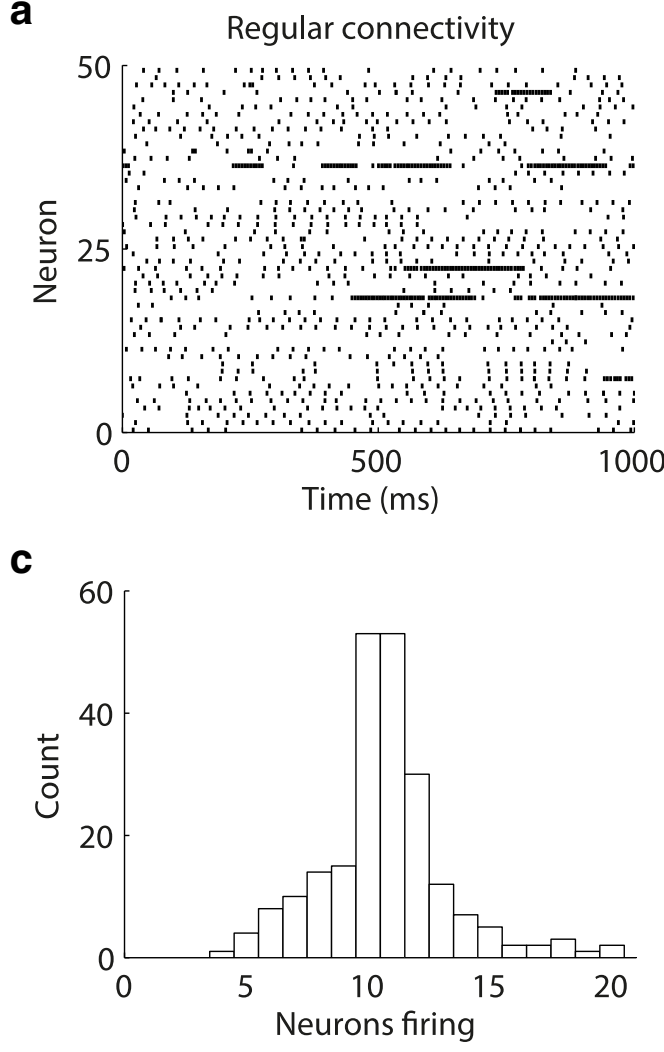

e

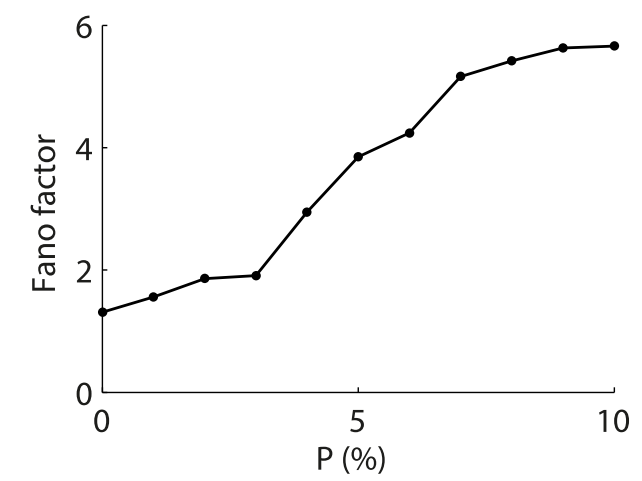

b

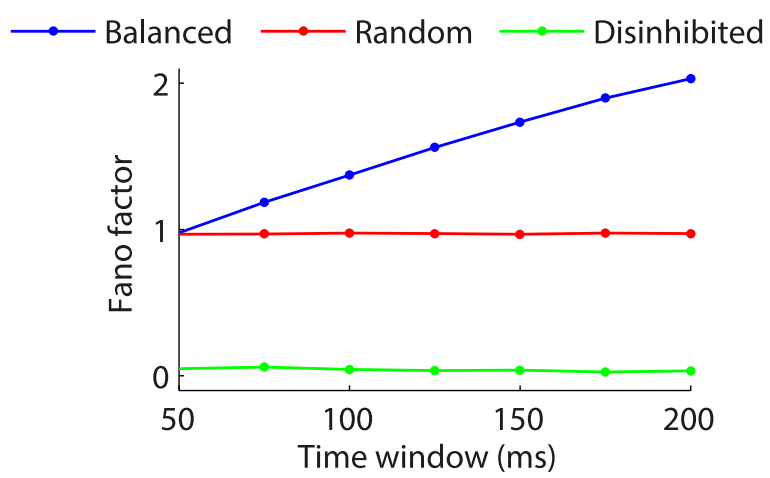

d

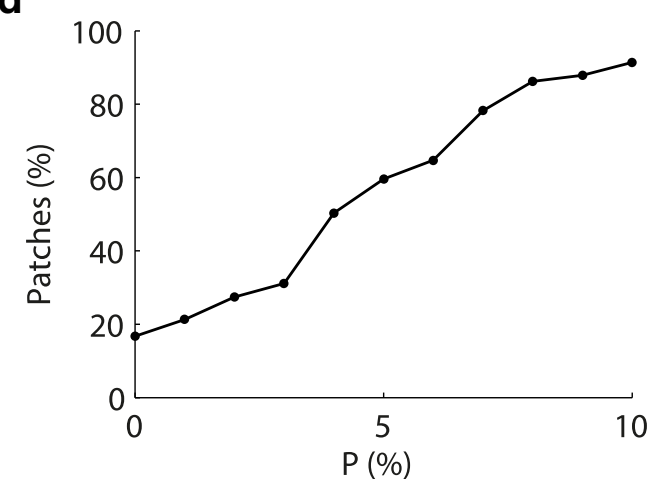

f

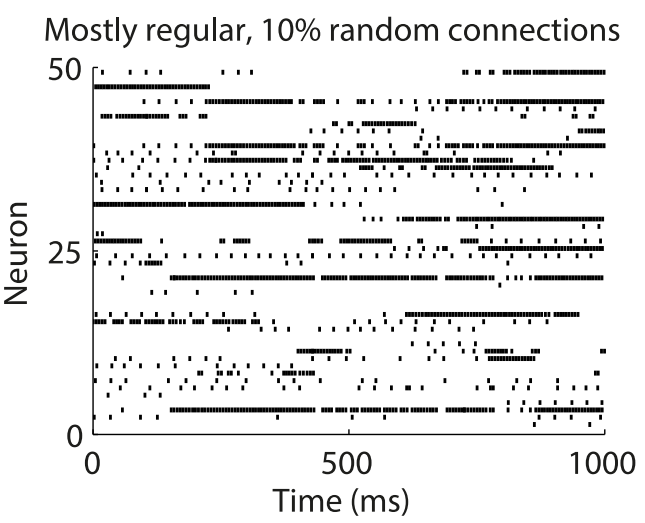

Figure 3. Properties of the spiking activity of network neurons. $\boldsymbol{a}$, Raster plot showing the spike times of a subpopulation of 50 excitatory neurons. Firing activity with variable spike timing is punctuated by dynamic transitions between high and low firing activity states. $\boldsymbol{b}$, For the balanced, spatially extended network, Fano factors increase as a function of length of time window (blue line). The neurons in the balanced, randomly connected network (red line) have a high Fano factor, but it is independent of time window. For the disinhibited network (green line), the Fano factor is independent of time window and has a value of almost zero. $c$, Histogram of the number of neurons in a $5 \times 5$ subgrid that would fire when there is a crescent-shaped wave passing by the subgrid. $\boldsymbol{d}$, Percentage of patchy patterns as a function of random rewiring probability $P$. In the balanced, spatially extended network, the percentage of patchy patterns increases with $P$. $\boldsymbol{e}$, Average Fano factor as a function of random rewiring probability $P$. In the balanced, spatially extended network, the Fano factor increases with $P$. $f$, Raster plot showing the spike times of a subpopulation of 50 excitatory neurons in the spatially extended network with $10 \%$ of the connections randomly rewired.

fraction of neurons in a grid to fire, their passage corresponds to the relatively low-activity state. This can be illustrated by choosing a neuron that has transitions between the low and highactivity states, and we find that, during the time periods when the neuron is not within a patchy pattern and crescent waves occasionally pass the location of the neuron, the mean firing rate of the neuron is $16 \mathrm{~Hz}$. On the other hand, when a patchy pattern moves to this location, the pattern wanders around the location due to its normally diffusive dynamics, and drifts away slowly; this enables the neuron within that location to fire persistently, corresponding to the high-activity state as shown in Figure $3 a$. For the time periods when the example neuron is within a patchy pattern, we find that the mean firing rate is $137 \mathrm{~Hz}$, and similar values can be found for other neurons, which are passed by patchy patterns. The low-activity state is therefore associated with crescent-shaped waves, whereas the transition to the highactivity state is caused by the approach of a patchy pattern.

Based on the above result, one would expect that the time scale of these transitions is closely related to the density of the patchy patterns. To test this prediction, we randomly rewire connections in the network with a certain probability $P$ (see Materials and Methods). Despite the random connections, the localized patterns shown in Figure $1 b-d$ can still emerge for $P \leq 10 \%$. Figure $3 d$ shows that, for the network with random connections, the 
a

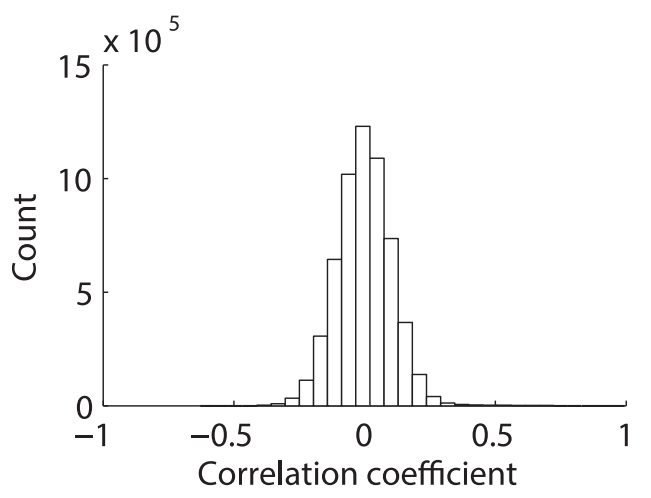

C

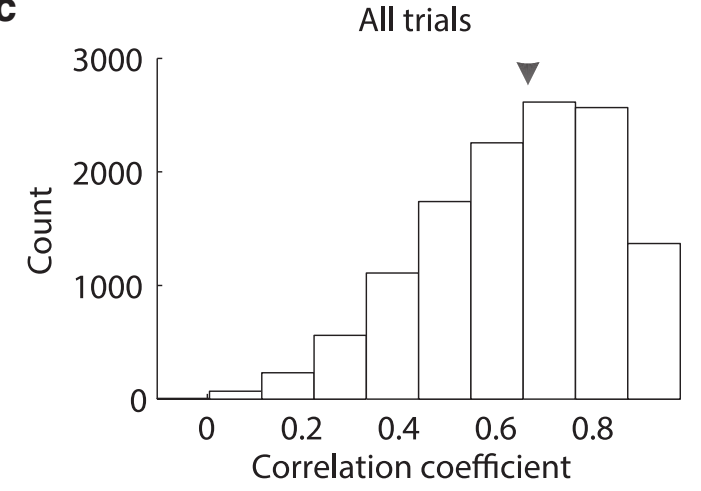

e

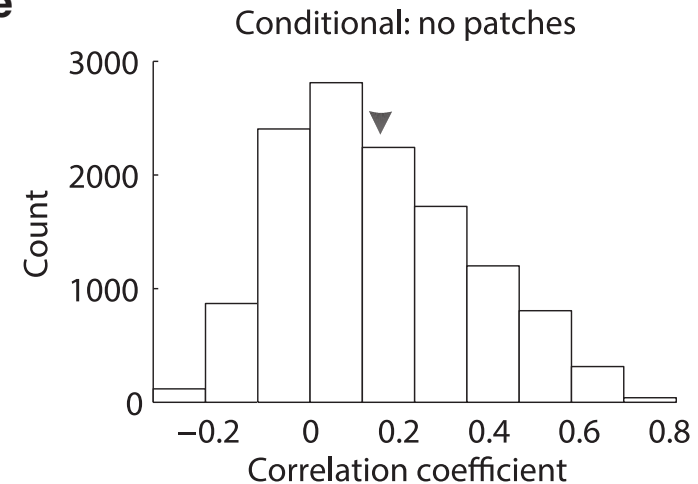

b

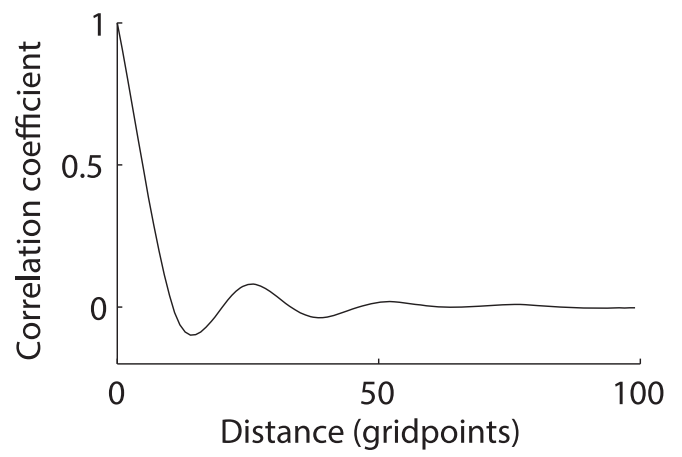

d

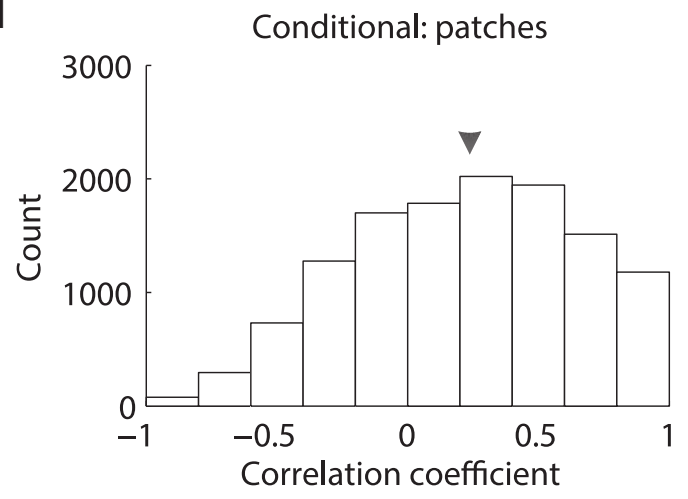

Figure 4. Spike count correlations between pairs of neurons in the balanced, spatially extended network. $\boldsymbol{a}$, Histogram of the correlation coefficients of random pairs of neurons; it is wide and centered around zero. $\boldsymbol{b}$, Average correlation coefficients, computed over all neuron pairs, as a function of their distance. c, Histogram of spike count correlations for nearby neurons (separated by 5 gridpoints), which has a mean value of 0.66 , as indicated by the arrow. $\boldsymbol{d}$, Histogram of spike count correlations for nearby neurons (separated by 5 gridpoints) when they are in the high-activity state; the mean value is 0.22 , as indicated by the arrow. $\boldsymbol{e}$, Histogram of spike count correlations for nearby neurons (separated by 5 gridpoints) when they are in the low-activity state; the mean value is 0.15 , as indicated by the arrow.

proportion of patchy patterns relative to the number of crescentshaped waves is greater than that in the original model; indeed, the proportion increases monotonically as the rewiring probability $P$ increases. This increase in the proportion of patchy patterns results in an increase in Fano factors (Fig. 3e). This is because the greater the proportion of the patchy patterns, the greater the frequency of transitions between high and low-activity states, as shown in Figure $3 f$. The Fano factor asymptotically approaches a particular value, which corresponds to an absence of the crescentshaped waves. Because the crescent-shaped waves are mostly responsible for the low-activity state, their absence means that neurons exhibit high firing rates or no firing at all; this results in greater fluctuations of firing rates.

To show that the propagating wave patterns emerging from the balanced network are essential for doubly stochastic firing activity, we calculate the Fano factors of the unbalanced network and the random network. The global ring, plane, and spiral waves (Fig. 1e,h), which occur in the unbalanced network, cannot cause variable spike timing. In this case, the Fano factors of firing activity are not only independent of time windows but also have very low values (Fig. $3 b$, green line). The randomly coupled network, which cannot generate any structured patterns (Fig. 1a), can produce spike count variability, but it does not exhibit slow fluctuations in firing rates; as shown in Figure $3 b$, whereas its Fano factor is close to 1, it is independent of time window.

Previous models of balanced networks have shown that averaged spike count correlations of pairs of cortical neurons form a wide distribution (i.e., with a high SD) with a mean value close to zero (Renart et al., 2010; Litwin-Kumar and Doiron, 2012). We calculate the correlation coefficients (see Materials and Methods) of spike counts of pairs of randomly selected neurons in the balanced, spatially extended network. Figure $4 a$ shows a wide distri- 
bution of correlations with a mean of $0.0008 \pm 0.0002$, similar to that found in Litwin-Kumar and Doiron (2012). We also calculate the correlation coefficients as a function of distance (Fig. 4b): at relatively short distance, there are positive correlations that decrease as the distance increases, which is largely consistent with the distance-dependent neuronal correlations as observed in Smith and Kohn (2008) and Ecker et al. (2014). The correlations are negative at intermediate distances and approach zero when distance is large enough; this is consistent with the negative correlations found for the randomly selected neuron pairs (Fig. 4a) (Renart et al., 2010; Ecker et al., 2014). Negative correlations have also been observed by Vaadia et al. (1995).

As illustrated above, there are transitions between low- and high-activity states, with the latter being produced by the slowly propagating patchy patterns. That is, when a patchy pattern moves to a location in the network, it drifts away from the location slowly. This makes the neurons within the location maintain sustained firing states for a relatively long period of time, resembling the "up state" found in the cortex. To show whether the correlations depend on the states of neuron firing activity for nearby neurons, as observed in Ecker et al. (2014), we also calculate the distribution of spike count correlations when patchy patterns are over a neuron pair (high-activity state), and when they are not (low-activity state); these are then compared with those obtained over all trials. To do this, we classify the high firing state as those $\Delta t=50 \mathrm{~ms}$ intervals with spike counts corresponding to a firing rate of $>50 \mathrm{~Hz}$. The mean values of the spike count correlations are $0.66,0.22$, and 0.15 for all trials (Fig. $4 c$ ), for the case when both neurons are in a high firing state (Fig. $4 d$ ), and for the case when they are not (Fig. 4e), respectively. The mean value of the correlation coefficients of the high-activity state is significantly larger than that of the low-activity state $(p<0.001)$, and both are significantly smaller than that for all trials $(p<0.001)$. These results therefore indicate that, in our model, the spike count correlations depend on the states of neural firing activity.

In total, the results we have obtained show that the balanced, spatially extended network with emergent propagating wave patterns is able to capture salient characteristics of variable firing activity of cortical neurons, including their variable spike timing, slow fluctuations of firing rates, small spike count correlations of randomly selected neuron pairs, and state-dependent spike count correlations.

\section{Synchronized synaptic inputs to individual neurons}

In the input synchrony model proposed for explaining variable neural dynamics, synaptic inputs to individual neurons consist of large, brief, and occasional inputs or "bumpy" inputs, indicating the synchronous arrival of many spikes of presynaptic neurons (Stevens and Zador, 1998; Okun and Lampl, 2008). These kinds of synchronized bumpy synaptic inputs have been found in the cortex (Hasenstaub et al., 2005; DeWeese and Zador, 2006; Okun and Lampl, 2008; Poulet and Petersen, 2008; Tan et al., 2014), but the mechanisms that give rise to such inputs have not been studied. We now show that, in our balanced, spatially extended network, the emergent propagating wave patterns provide a network mechanism to generate such inputs.

Figure $5 a$ shows the dynamics of the excitatory synaptic conductance received by individual neurons in our model, which are characterized by quiescent periods punctuated by large, brief, and occasional excursions in amplitude, with variable magnitudes and shapes. Figure $5 b$ shows the distribution of the excitatory synaptic conductance $g_{i j}^{E}(t)$ received by each neuron over the whole time course of one trial; the distribution has a heavy tail.
We use a linear-log plot because we can use it to directly compare with empirical observations in DeWeese and Zador (2006) and Hromádka et al. (2013), but it is evident that the distribution is heavy-tailed if the scale of both axes is linear. It is interesting to note that heterogeneous synaptic inputs with heavy-tail distributions have been found in the somatosensory cortex (Lefort et al., 2009). To quantify these non-Gaussian dynamics, we calculate the kurtosis for the distribution of the excitatory synaptic conductance of each neuron within the network (see Materials and Methods). We find that these values display a wide range of activity with a maximum kurtosis of 17.6 and a minimum kurtosis of -1.1 . However, negative values of kurtosis are rare; most values are positive, with a population mean of 5.9 (SD 1.6). These kurtosis values are comparable with those implied by whole-cell recordings of auditory cortex in awake rats (Hromádka et al., 2013). Because the kurtosis is a measure of the proportion of samples within the peak and tail of a distribution compared with a normal distribution, the kurtosis is positive for heavy-tail distributions (see Materials and Methods). The negative values of kurtosis indicate that distributions of the inputs may occasionally be bimodal; such distributions may result from periods of quiescence punctuated by periods of sustained input.

We next illustrate that the dynamics of propagating wave patterns in our network provide a mechanistic explanation for the bumpy, synchronized synaptic inputs, as shown in Figure $5 a$. It is apparent that a wave front, which includes multiple spiking neurons, provides a source of synchronized input to any neurons that it approaches. However, the superdiffusive dynamics of the crescent-shaped waves means that such synchronized input is transient, as the wave will pass by and move out of range of the neuron rapidly. We can quantify the number of spikes participating in a bumpy synaptic input by considering a planar wave front approaching a "test" neuron. The maximum distance of excitatory connections is $D^{E}=10$; above this distance, the test neuron will not receive any recurrent input (see Materials and Methods). Therefore, we assume the initial displacement between the wave and the test neuron to be $d=-D^{E}$. If the wave approaches the test neuron head-on, the neuron will continue to receive input until the wave passes out of range at $d=D^{E}$. Because $d$ is in units of neuron separation (i.e., gridpoints), the test neuron receives excitatory inputs from the wave front (i.e., at $2 D^{E}+1$ locations); this is the range over which the wave provides input to our test neuron as it approaches and then moves away; in other words, it is the length of the wave trajectory that affects the test neuron. The width of a typical wave front consists of $N_{w} \approx 19$ (Fig. 1i) depolarized neurons. The number of neurons that could participate in a bump of synaptic input is the width multiplied by the length of the trajectory (i.e., $\left.N_{w}\left(2 D^{E}+1\right)-1\right)$, where the minus one excludes the test neuron from receiving input from itself. Excluding neurons, which are separated from the test neuron by a distance greater than $D^{E}$, we find that 315 neurons can participate in the bump. This is similar to the number of synthetic excitatory postsynaptic potentials used to generate synchronized inputs to cortical neurons in brain slices (Stevens and Zador, 1998), but it is approximately an order of magnitude less than that found in vivo (DeWeese and Zador, 2006). This discrepancy is likely due to the scaling of our network (see Materials and Methods).

The model of a typical wave front approaching a test neuron can also be used to explain the shape of the bumps of synaptic conductance. Consider the wave front moving at the average speed of 2 gridpoints/ms (Fig. $2 c$ ) head-on toward a test neuron and then away from it. As excitatory coupling strength has an exponential dependence on distance (Eq. 4, see Materials and 
a

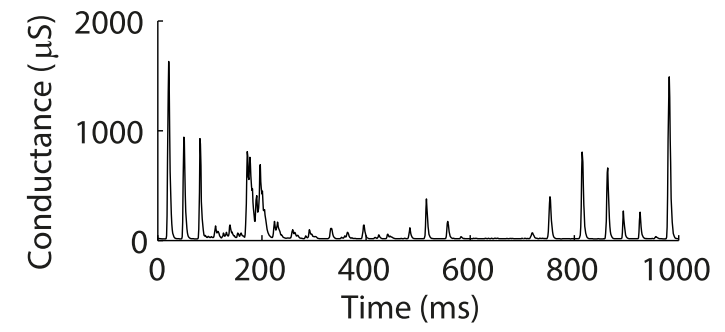

C

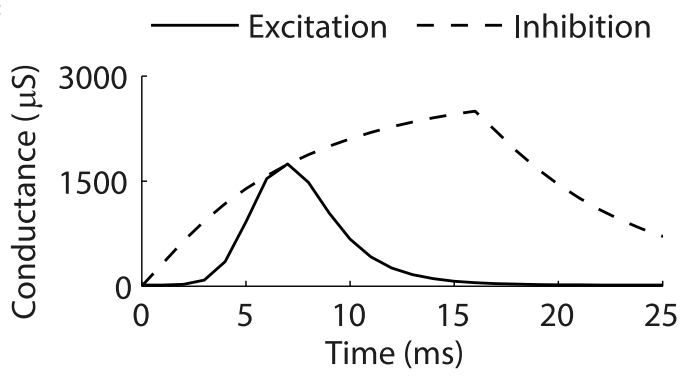

e

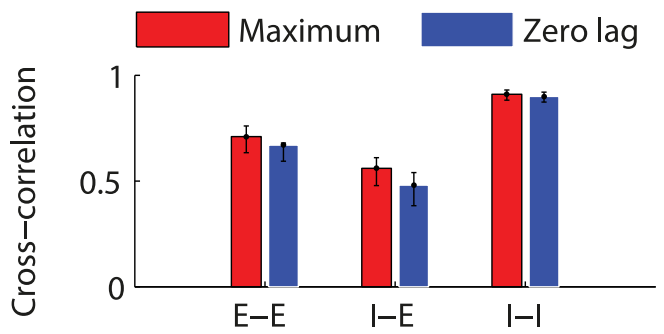

g

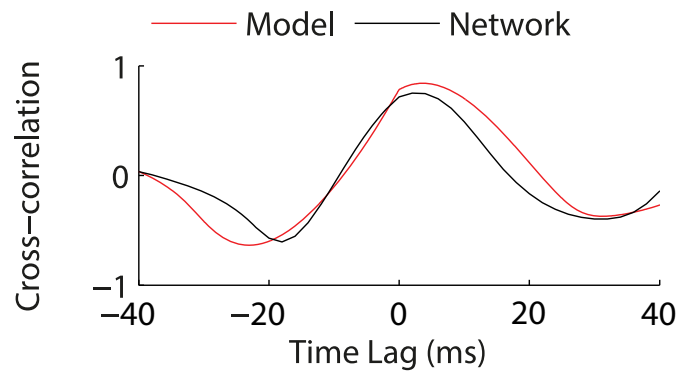

b

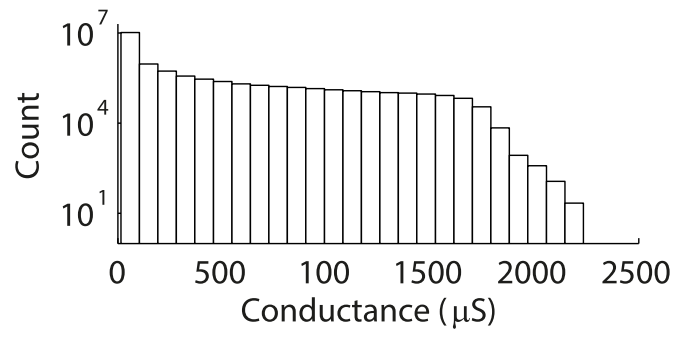

d

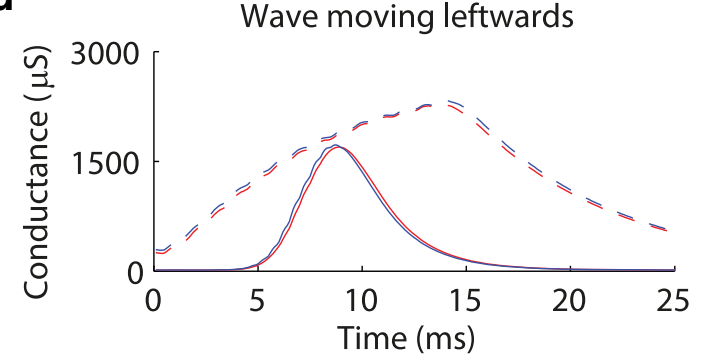

f

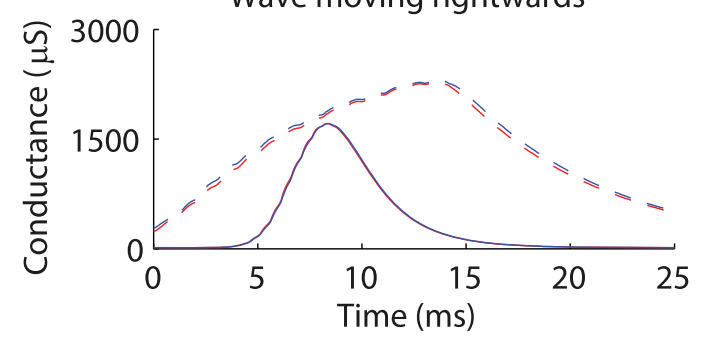

Figure 5. Properties of synaptic inputs to neurons in the balanced, spatially extended network. $\boldsymbol{a}$, Time series of excitatory synaptic conductance $g_{i j}^{E}(t)$ received by a neuron over $1 \mathrm{~s}$; it consists of quiescent periods punctuated by brief excursions in conductance, indicating the arrival of highly synchronized inputs of many neurons. The time courses of $g_{i j}^{E}(t)$ have variable shape and duration; for instance, at $\sim 200 \mathrm{~ms}$, the bump in synaptic conductance has a relatively longer duration. $\boldsymbol{b}$, Histogram of excitatory synaptic conductance $g_{i j}^{E}(t)$ received by each neuron over the whole time course of one trial (i.e., the trace of the conductance as shown in $\boldsymbol{a}$ ). The bumpy synaptic input results in a heavy-tail distribution of conductances. Note the logarithmic scale of the coordinate; this is used for direct comparison with relevant empirical data, but the heavy tail is still evident for a plot with both axes linearly scaled. $c$, Excitatory (solid line) and inhibitory (dashed line) inputs received by a test neuron in the model of a wave front of width $N_{w} \approx 19$ approaching a test neuron head-on. $\boldsymbol{d}$, The conductances received by two nearby neurons in the network, shown as red and blue lines, as a wave passes by. Solid lines indicate excitatory conductance. Dashed lines indicate inhibitory conductance. $\boldsymbol{e}$, Average cross-correlation of synaptic inputs received by neuron pairs separated by an interneuron distance of 5 . There are high cross-correlations of 0.69 (SD 0.04) for excitatory-excitatory (E-E), 0.56 (SD 0.05) for inhibitory-excitatory (I-E), and 0.91 (SD 0.02) for inhibitoryinhibitory (I-I) conductances, respectively. Red bars represent maximum cross-correlation. Blue bars represent cross-correlation at zero time lag. $f$, The conductances received by two nearby neurons in the network as a wave approaches them from the opposite direction to that for $\boldsymbol{d} . \boldsymbol{g}$, Cross-correlation between the inhibitory input and the excitatory input found for nearby neurons in the network (black line) and the cross-correlation calculated for the model of a wave approaching a test neuron (red line).

Methods), the amplitude of the excitatory inputs first increases and then decreases. The resultant time course of the input produced in this model is similar to the time course (full width at half-maximum is $4.4 \mathrm{~ms})$ and amplitude $(\sim 2000 \mu \mathrm{S})$ of the bumps of largest amplitude in the network model (Fig. $5 c$, solid line). Bumpy synaptic inputs in our network usually have diverse profiles, but such variations can arise from differences in the sizes of firing wave fronts and the complex trajectories of these waves, which often do not approach a neuron head-on but move within its proximity. In summary, the crescent-shaped waves with su- perdiffusive dynamics can produce bumpy, synchronized inputs with short durations, to individual neurons.

Bumpy synaptic inputs in the balanced, spatially extended network occasionally have longer durations. For instance, the bumpy synaptic conductance centered around $200 \mathrm{~ms}$ in Figure $5 a$ has a relatively longer duration, during which the neuron receives synchronized inputs for a sustained period (i.e., up states), similar to intracellular recordings in the auditory cortex of awake rats (Hromádka et al., 2013). These inputs are caused by patchy patterns because, when these patterns move toward a par- 
ticular neuron, they wander around the neuron and drift away from it slowly in a normally diffusive manner, providing relatively sustained synchronized inputs to the neuron. To further quantify the bumpy, synchronized synaptic inputs, we study their durations, which we define as the time intervals during which excitatory conductance exceeds some threshold. We choose this threshold to be the leak conductance (i.e., $g_{i j}^{E}(t)>g_{L}$ ), but as long as the threshold level is not too small, its precise value is not critical; choices near this value yield similar results. Using this definition, we classify bumpy inputs with long duration as those of duration $>500 \mathrm{~ms}$, as was used by Hromádka et al. (2013). We find that bumpy inputs of long duration are rare, comprising only $0.05 \%$ of all bumps. Furthermore, most time spent away from rest is spent in short bumps; only $1.2 \%$ of total simulation time is spent in bumpy inputs with duration $>500 \mathrm{~ms}$. Indeed, bumpy inputs of long duration constitute $3.6 \%$ of the duration of all bumpy, synchronized inputs, despite, on average, being approximately an order of magnitude longer than bumpy inputs of short duration (Fig. 5a). This is in agreement with experimental results, which suggest that sustained bumps (up states) in inputs are rare in the awake cortex (Hromádka et al., 2013).

\section{Synaptic inputs are correlated and inhibition lags behind excitation}

We now demonstrate that our model is also able to capture the cross-correlations of synaptic inputs to pairs of nearby neurons, as measured by using dual-cell recording in Okun and Lampl (2008). Figure $5 d$ shows the typical excitatory (solid lines) and inhibitory (dashed lines) conductances received by two neurons separated by a small distance of 5 gridpoints, as a wave passes by; conductance is positive (Eq. 2 in our model). We calculate the cross-correlations of these inputs over pairs of neurons and over trials and find that there are large peak values of cross-correlation with 0.69 (SD 0.04) for excitatory-excitatory, 0.56 (SD 0.05) for inhibitory-excitatory, and 0.91 (SD 0.02) for inhibitoryinhibitory conductances, respectively (Fig. 5e). This is comparable with the cross-correlations of excitatory and inhibitory inputs found in nearby cortical neurons (Okun and Lampl, 2008; Gentet et al., 2010).

As shown in Figure $5 d$, excitatory inputs to the nearby neurons reach their peak values at almost the same time; this is also the case for the inhibitory inputs. In contrast, inhibition lags excitation significantly $(p<0.001)$. As in Okun and Lampl (2008), such lags between inhibition and excitation are then measured based on the peak values of their cross-correlations averaged over neuron pairs and trials; these cross-correlations attain their peak value at non-zero lag, which is $2.3 \pm 0.4 \mathrm{~ms}$. This lag is comparable to the lag of $3.8 \pm 4.9 \mathrm{~ms}$ as found in Okun and Lampl (2008). We also calculate the cross-correlation of excitatory and inhibitory conductances arriving at the same neuron and obtain a lag of $2.2 \pm 0.5 \mathrm{~ms}$, comparable with the lag of $2.4 \pm$ $3.6 \mathrm{~ms}$ reported by Wehr and Zador (2003) for such inputs received by the same neuron. Our model is therefore able to reproduce a characteristic feature observed in cortical neurons, namely, that inhibition lags behind excitation by several milliseconds. This supports the hypothesis that inhibition controls the integration time window of excitation, enabling neurons to act as coincidence detectors.

In our model, the bumpy excitatory synaptic inputs are always ahead of inhibitory inputs, as shown in Figure $5 d$, regardless of which direction the approaching wave moves; Figure $5 f$ shows such inputs when there is a wave moving in the opposite direction to that in Figure $5 d$. This effect is a result of the distance- dependent coupling and the distinct synaptic time scales of excitatory and inhibitory dynamics $\left(\tau_{d}^{E}=2.0 \mathrm{~ms}\right.$ and $\tau_{d}^{I}=7.0 \mathrm{~ms}$ in Eq. 3). To illustrate this, we consider the model mentioned earlier, of a propagating wave front consisting of $N_{w}=19$ firing excitatory neurons, and extend it to include inhibitory neurons. Because inhibitory neurons only constitute $20 \%$ of the neurons in the network, there are half as many inhibitory neurons as there are excitatory neurons along both dimensions. Therefore, the firing wave front consists of $\sim N_{w} / 2$ inhibitory neurons, which occur at $d=-D^{I}-D^{I}-2, \ldots,-1,1, \ldots, D^{I}-2, D^{I}$, where $D^{I}=15$ is the spatial range of inhibitory neurons. Using this extended model, we obtain the time course of inhibitory input received by our test neuron, shown by Figure $5 c$ (dashed line); this is similar to what is observed in our balanced network (Fig. $5 d, f)$. When we calculate the cross-correlation between excitatory and inhibitory bumps, we find that it obtains its peak value at $3.9 \mathrm{~ms}$ (Fig. $5 g$, red line), similar to the lag found when a wave passes a neuron in the balanced, spatially extended network (Fig. $5 g$, black line). Based on the model of a wave front approaching a test neuron, we also find that the time lag remains the same for a wave moving in an opposite direction (i.e., starting at $d=D^{I}$ and ending at $d=-D^{I}$ ). However, if we run the model with the same synaptic time scales $\left(\tau_{d}^{E}=2.0 \mathrm{~ms}\right.$ and $\left.\tau_{d}^{I}=2.0 \mathrm{~ms}\right)$, no lag exists in the cross-correlation function; if we run the model with the same distance-dependent coupling for excitatory and inhibitory neurons $\left(K_{i j, i^{\prime} j^{\prime}}^{E}=K_{i j, i^{\prime} j^{\prime}}^{I}\right.$ in Eq. 4), the lag is only $2.3 \mathrm{~ms}$. These results therefore suggest that the lags between excitation and inhibition are dependent on the interplay of the synaptic time scales and the distance-dependent coupling but are independent of the propagation direction of the wave patterns. We also find that this lag is not evident for the balanced, randomly connected network, in which no wave patterns can form.

\section{Membrane potential dynamics are non-Gaussian}

In previous models with balanced excitation and inhibition, membrane potential follows a random walk and its distribution is Gaussian (Shadlen and Newsome, 1998); this is also the case for our randomly connected network, in which the membrane potential has a Gaussian distribution with a kurtosis near zero. However, for the balanced, spatially extended network, the synchronized bumpy inputs cause occasional large excursions of membrane potential from rest; this results in a heavy-tail distribution of membrane potential (Fig. 6a). This non-Gaussian dynamic is confirmed by calculating the kurtosis, which has a mean value over all neurons of 5.9 (SD 1.2), close to the kurtosis found in DeWeese and Zador (2006). The distribution of kurtosis values for the membrane potential of each neuron shows that there is a great diversity in membrane potential dynamics, with a maximum kurtosis of 11.8 and a minimum kurtosis of -0.3 (Fig. 6b). We find similar distributions for the synaptic inputs (Fig. 5b), which indicates that there are bumpy dynamics for both synaptic input and membrane potential. This coincides with the passing of waves, which causes highly synchronized synaptic inputs and a resultant sudden increase in membrane potential.

Finally, we consider the correlation properties of membrane potential. We calculate the auto-correlation of the membrane potential of each neuron and then calculate the average. As shown in Figure $6 c$, there are oscillations in auto-correlation as a function of time lag with a frequency of $33 \mathrm{~Hz}$. Applying the same procedure to the excitatory synaptic inputs received by each neuron (Fig. $6 d$ ) yields a similar auto-correlation, with a characteristic frequency of $33 \mathrm{~Hz}$. We also calculate the cross-correlation of the membrane potentials of nearby neurons. The averaged cross- 
a

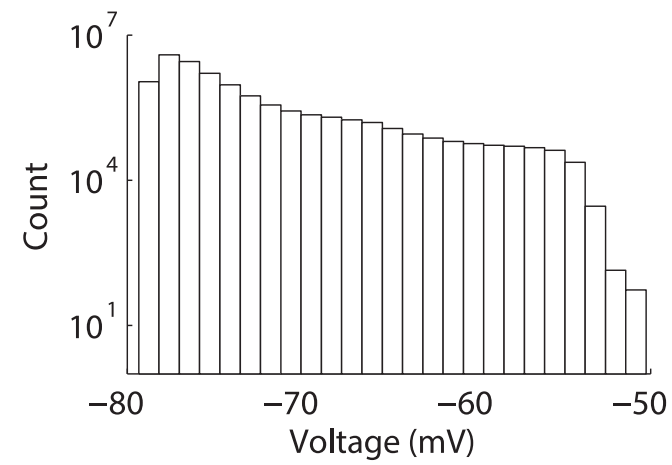

C

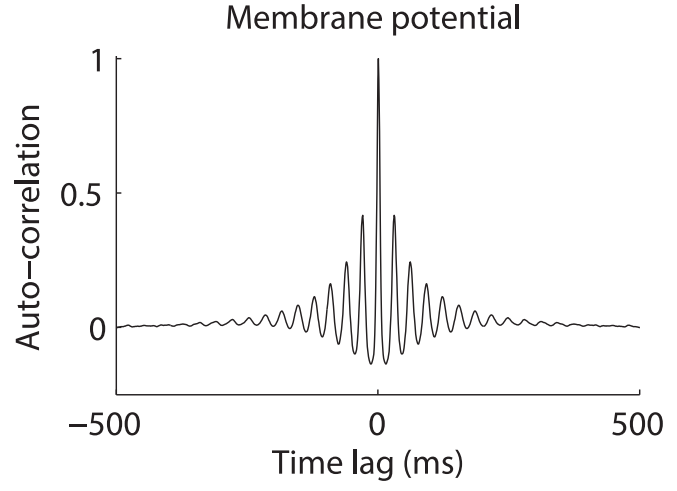

e

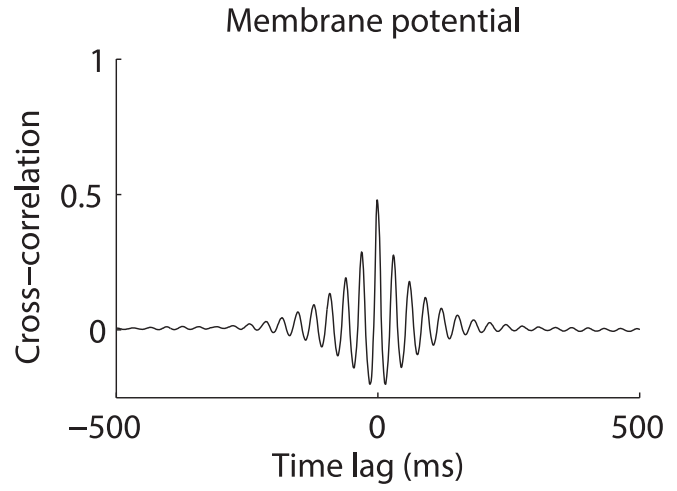

b

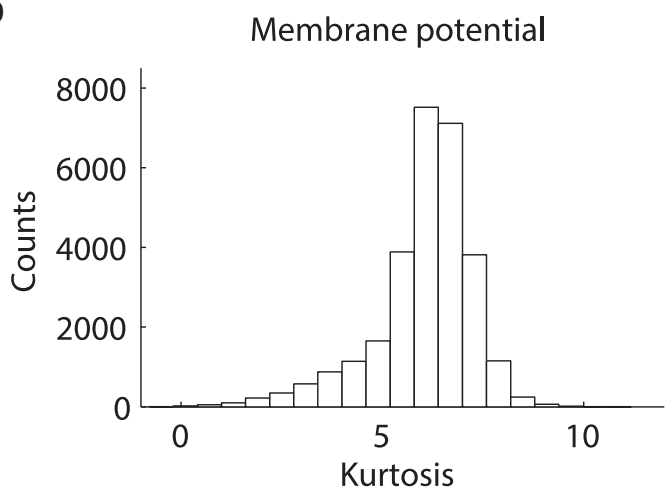

d

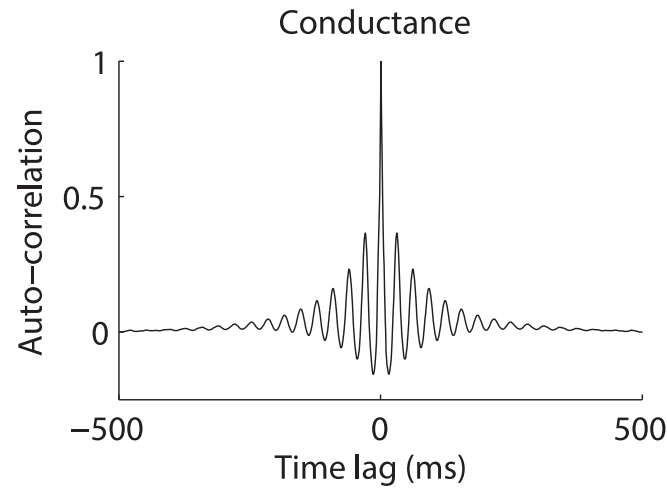

Figure 6. The dynamics of membrane potential in the balanced, spatially extended network. $\boldsymbol{a}$, Distribution of the membrane potential of all neurons over the time course of one trial is heavily tailed. Note the logarithmic scale of the coordinate; this is used for direct comparison with empirical data, but the heavy tail is still evident if the scale of both axes is linear. $\boldsymbol{b}$, Distribution of kurtosis values of membrane potentials of each neuron for all trials. $c$, Average auto-correlation of membrane potential of neurons within one trial. It has a frequency of $33 \mathrm{~Hz}$, and the envelope of the auto-correlation decays as time lag increases. $\boldsymbol{d}$, Average auto-correlation of excitatory synaptic inputs received by neurons within one trial. It has a frequency of $33 \mathrm{~Hz}$, and the envelope of the auto-correlation decays as time lag increases. $\boldsymbol{e}$, Average cross-correlation of membrane potential of pairs of nearby neurons within one trial. It also has a frequency of $33 \mathrm{~Hz}$, and it decays with time lag but has a smaller amplitude (maximum cross-correlation is 0.5 ).

correlation has the same frequency as the averaged autocorrelation but has a smaller amplitude of 0.5 , as shown in Figure $6 e$; this is due to the dependence of excitatory connection strength upon distance (Eq. 4, see Materials and Methods). These correlated functions of membrane potential produced in our model are similar to those found in previous experiments of awake animals (Poulet and Petersen, 2008; Gentet et al., 2010). The frequency is, however, an order of magnitude greater than that found experimentally (Poulet and Petersen, 2008; Gentet et al., 2010), a discrepancy that is likely due to the scale of our network (see Materials and Methods). Suppose that the speed of the wave patterns is $v$ and the spatial separation between them is $l$; then on average, the interval between waves approaching a certain area would be $l / v$. The corresponding frequency of synaptic inputs to the neurons around the area would therefore be $v / l$, meaning that increasing wave separations can po- tentially decrease the frequency. Indeed, when the network is scaled up to $600 \times 600$ with larger synaptic coupling ranges $\left(D^{E}=20\right.$ and $D^{I}=30$ in Eq. 4), we find that the average frequency decreases from $33 \mathrm{~Hz}$ to $21 \mathrm{~Hz}$.

\section{Discussion}

In this study, we have shown that propagating wave patterns with complex dynamics can emerge from spatially extended, spiking neural circuits with balanced excitation and inhibition. These patterns provide a mechanistic explanation for a range of neural dynamics, including the variability of spike timing, slow fluctuations of firing rates, and non-Gaussian dynamics of membrane potential. Additionally, these propagating wave patterns enable individual neurons to have highly synchronized synaptic inputs, as found previously (Stevens and Zador, 1998; DeWeese and Za- 
dor, 2006; Okun and Lampl, 2008). Our model therefore reconciles the model of balanced excitation and inhibition, and that of synchronized inputs, to account for irregular neural dynamics.

\section{A unified account of irregular neural dynamics}

Neural firing activity in vivo can be approximated as a doubly stochastic process (Churchland et al., 2011) because, in addition to the variability of spike timing, the firing rates of cortical neurons fluctuate over long time scales. A recent study, by extending conventional balanced networks with random uniform connections to those with clustered connections, is able to explain such doubly stochastic processes (Litwin-Kumar and Doiron, 2012). As in Litwin-Kumar and Doiron (2012), our model exhibits slow firing rate fluctuations while retaining the fast spiking variability. In Litwin-Kumar and Doiron (2012), the mechanism underlying the fluctuations of firing rates is spontaneous switching between two attractors, with one attractor representing the low-activity state and the other one representing the high-activity state. However, as we have demonstrated in our model, firing rate fluctuations are caused by the complex collective dynamics of propagating wave patterns, including their normally diffusive and superdiffusive dynamics. The dynamics of membrane potential, as found by whole-cell recordings, impose another set of constraints for theoretical accounts of variable neural activity, including their correlated fluctuations (Poulet and Petersen, 2008; Gentet et al., 2010) and non-Gaussian distributions (DeWeese and Zador, 2006; Hromádka et al., 2013; Tan et al., 2014). These important empirical observations have not been addressed in previous modeling studies but are captured by our model.

Our propagating wave-based model is able to reconcile the view of balanced excitation and inhibition and that of synchronized inputs to account for variable neural dynamics. On the one hand, propagating wave patterns with complex dynamics only emerge when excitatory and inhibitory synaptic currents are balanced. On the other hand, the emergent wave patterns provide individual neurons with large, synchronized inputs. In our model, when a propagating wave pattern moves toward a neuron, this neuron receives synaptic inputs from the firing neurons within the pattern. However, this pattern eventually leaves that neuron, resulting in a bump of large synaptic inputs. Because these patterns move in a seemingly random way, they produce synchronized, bumpy inputs that are randomly distributed in time for individual neurons. Such inputs have been synthesized and injected into cortical neurons in brain slices to account for the variability of spike timing (Stevens and Zador, 1998) and have also been found in the auditory cortex (DeWeese and Zador, 2006; Hromádka et al., 2013) and the somatosensory cortex of rats (Okun and Lampl, 2008). Aside from unraveling the network mechanism of these synchronized inputs to individual neurons, our propagating wave-based model can reproduce the crosscorrelations of excitatory and inhibitory synaptic inputs to nearby neurons, and the time lag of several milliseconds between them, as observed in Okun and Lampl (2008).

In our model, the synchronized synaptic inputs cause nonGaussian membrane potential dynamics, consisting of quiescent periods that are interrupted by short intervals of high-amplitude depolarization. Such non-Gaussian fluctuations of membrane potential have been observed in cortical neurons (DeWeese and Zador, 2006; Tan et al., 2014) but are inconsistent with the existing random walk models (Shadlen and Newsome, 1998) and the existing balanced network models (van Vreeswijk and Sompolinsky, 1998), which instead predict Gaussian dynamics of membrane potential. The main mechanism of the non-Gaussian dynamics of membrane potential in our model is the synchronized synaptic inputs with a heavy-tail distribution, indicating that synaptic inputs have heterogeneous magnitudes instead of homogeneous ones. Although the classical random walk model has served as a simple but powerful framework to understand the variability of neural dynamics, our study suggests a significant extension, in which balanced synaptic inputs with heterogeneous magnitudes should be considered.

\section{Propagating wave patterns in balanced, spatially extended neural networks}

The balanced networks studied here consist of conductancebased, integrate-and-fire neurons that are uniformly distributed over a 2D lattice. A key feature considered in these models is that the neurons, particularly excitatory neurons, receive inputs from neurons that are in physical proximity to them and that coupling strengths decrease as distance between neurons increases. Such a distance-dependent rule has been found in the connections of neurons and those of interareal networks; this coupling rule was recently hypothesized to be a general design principle applying over multiple scales of neural systems (Ercsey-Ravasz et al., 2013). Inhibitory coupling, however, is homogeneous, in accord with other modeling studies (Litwin-Kumar and Doiron, 2012); such homogeneous inhibitory connectivity, with less tuning specificity than that of excitatory neurons, has been reported in experimental studies (Fino and Yuste, 2011). We have mainly studied the intrinsic dynamics of propagating wave patterns that emerge from the network with regular structure and constant external inputs. However, we have found that such emergent dynamics are still preserved when some connections of the network are randomly rewired and when neurons receive noisy inputs. Another key feature in our network is that it is 2D; for the randomly coupled version of our model, we have shown the network only generates variable spike trains, but it cannot produce the non-Gaussian dynamics of membrane potential and highly synchronized synaptic inputs, mainly due to a lack of emergent propagating waves. We have studied similar networks with a ring structure and found no evidence of such wave patterns with complex dynamics, which suggests that 2D spatial extension is important for the emergence of complex propagating wave patterns. This is similar to other complex physical systems where such spatial extension is essential for the emergence of complex spatiotemporal patterns (Bak et al., 1988).

Our work links the propagating wave patterns that have been widely observed in the cortex (Rubino et al., 2006; Benucci et al., 2007; Han et al., 2008; Wu et al., 2008; Sato et al., 2012) with variable neural dynamics. As demonstrated in our study, propagating waves exhibit complex dynamics: they originate from random locations and propagate in a seemingly random way. If the network is out of balance in the excitation dominated state, the network exhibits more regular, global wave patterns, such as spiral waves, as have been found in pharmacologically manipulated, disinhibited neural circuits (Huang et al., 2010); if the network is in an inhibition dominated state, these patterns disappear shortly after they are formed, without any long-range propagation. In our study, we have shown that the collective dynamics of crescent-shaped propagating waves in the balanced network is anomalous superdiffusion, suggesting that there are long-range correlations in wave pattern dynamics. This prediction can be tested by analyzing propagating waves found in experiments in the same way as we have done in our modeling study. 


\section{Computational implications of propagating wave patterns}

Our model shows that structured activity such as propagating wave patterns at the level of neural circuits can arise from highly variable firing activity of individual neurons, therefore bridging such seemingly contrasting dynamics at different neural levels. This property of spiking neural networks makes the cortex, a paradigmatic example of a complex system, analogous to other complex physical systems, such as turbulent fluids. In turbulence, coherent structures, such as vortices, similarly emerge from molecules that behave irregularly (Hussain, 1986). Our work, therefore, indicates that drawing on analogies between cortical circuits and turbulent fluids may bring novel insights into understanding complex spatiotemporal dynamics of the cortex (Wang, 2010).

What are the computational implications of the existence of propagating wave patterns in the cortex? Spiking waves, when propagating across neurons, would result in concerted synchronized volleys of activity from one subpopulation to the next, so that spike timing sequences can be formed. These sequences are an emergent property of our balanced networks rather than a product of propagation of synchronized volleys along feedforward networks, as in the framework of synfire chains (Abeles, 1991). Contrary to spike sequences propagating along feedforward chains with regular dynamics, the emergent spiking wave patterns in the balanced network have complex dynamics, including the presence of multiple, interacting waves distributed over space simultaneously. These dynamics may be necessary for neural systems to assemble different spiking sequences to achieve "neural syntax" (Buzsáki, 2010). The multiple, spatiotemporally distributed properties of localized propagating patterns also appear to be well suited for distributed parallel information processing; recently, it has been proposed that these patterns and their interactions can perform distributed dynamic computation in neural circuits (Gong and van Leeuwen, 2009). By showing that propagating wave patterns provide a unified account of a range of neural dynamics, our work suggests that it is important to explore their potential computational roles in the brain.

\section{Notes}

Supplemental material for this article is available at http://www.physics. usyd.edu.au/ gong (Video of Propagating Wave Patterns). This material has not been peer reviewed.

\section{References}

Abeles M (1991) Corticonics: neural circuits of the cerebral cortex. Cambridge: Cambridge UP.

Arieli A, Shoham D, Hildesheim R, Grinvald A (1995) Coherent spatiotemporal patterns of ongoing activity revealed by real-time optical imaging coupled with single-unit recording in the cat visual cortex. J Neurophysiol 73:2072-2093. Medline

Arieli A, Sterkin A, Grinvald A, Aertsen A (1996) Dynamics of ongoing activity: explanation of the large variability in evoked cortical responses. Science 273:1868-1871. CrossRef Medline

Bak P, Tang C, Wiesenfeld K (1988) Self-organized criticality. Phys Rev A 38:364-374. CrossRef Medline

Benucci A, Frazor RA, Carandini M (2007) Standing waves and traveling waves distinguish two circuits in visual cortex. Neuron 55:103-117. CrossRef Medline

Braitenberg V, Schüz A (1998) Cortex: statistics and geometry of neuronal connectivity. Berlin: Springer.

Britten KH, Shadlen MN, Newsome WT, Movshon JA (1993) Responses of neurons in macaque MT to stochastic motion signals. Vis Neurosci 10: 1157-1169. CrossRef Medline

Burger W, Burge MJ (2008) Digital image processing: an algorithmic introduction using Java. New York: Springer.

Buzsáki G (2010) Neural syntax: cell assemblies, synapsembles, and readers. Neuron 68:362-385. CrossRef Medline
Churchland AK, Kiani R, Chaudhuri R, Wang XJ, Pouget A, Shadlen MN (2011) Variance as a signature of neural computations during decision making. Neuron 69:818-831. CrossRef Medline

Churchland MM, Abbott LF (2012) Two layers of neural variability. Nat Neurosci 15:1472-1474. CrossRef Medline

Churchland MM, Yu BM, Cunningham JP, Sugrue LP, Cohen MR, Corrado GS, Newsome WT, Clark AM, Hosseini P, Scott BB, Bradley DC, Smith MA, Kohn A, Movshon JA, Armstrong KM, Moore T, Chang SW, Snyder LH, Lisberger SG, Priebe NJ, et al. (2010) Stimulus onset quenches neural variability: a widespread cortical phenomenon. Nat Neurosci 13:369378. CrossRef Medline

DeCarlo LT (1997) On the meaning and use of kurtosis. Psychol Methods 2:292-307. CrossRef

DeWeese MR, Zador AM (2006) Non-Gaussian membrane potential dynamics imply sparse, synchronous activity in auditory cortex. J Neurosci 26:12206-12218. CrossRef Medline

Ecker AS, Berens P, Cotton RJ, Subramaniyan M, Denfield GH, Cadwell CR, Smirnakis SM, Bethge M, Tolias AS (2014) State dependence of noise correlations in macaque primary visual cortex. Neuron 82:235-248. CrossRef Medline

Ercsey-Ravasz M, Markov NT, Lamy C, Van Essen DC, Knoblauch K, Toroczkai Z, Kennedy H (2013) A predictive network model of cerebral cortical connectivity based on a distance rule. Neuron 80:184-197. CrossRef Medline

Faisal AA, Selen LP, Wolpert DM (2008) Noise in the nervous system. Nat Rev Neurosci 9:292-303. CrossRef Medline

Ferezou I, Haiss F, Gentet LJ, Aronoff R, Weber B, Petersen CC (2007) Spatiotemporal dynamics of cortical sensorimotor integration in behaving mice. Neuron 56:907-923. CrossRef Medline

Fino E, Yuste R (2011) Dense inhibitory connectivity in neocortex. Neuron 69:1188-1203. CrossRef Medline

Gentet LJ, Avermann M, Matyas F, Staiger JF, Petersen CC (2010) Membrane potential dynamics of GABAergic neurons in the barrel cortex of behaving mice. Neuron 65:422-435. CrossRef Medline

Gong P, van Leeuwen C (2009) Distributed dynamical computation in neural circuits with propagating coherent activity patterns. PLoS Comput Biol 5:e1000611. CrossRef Medline

Han F, Caporale N, Dan Y (2008) Reverberation of recent visual experience in spontaneous cortical waves. Neuron 60:321-327. CrossRef Medline

Hasenstaub A, Shu Y, Haider B, Kraushaar U, Duque A, McCormick DA (2005) Inhibitory postsynaptic potentials carry synchronized frequency information in active cortical networks. Neuron 47:423-435. CrossRef Medline

Holt GR, Softky WR, Koch C, Douglas RJ (1996) Comparison of discharge variability in vitro and in vivo in cat visual cortex neurons. J Neurophysiol 75:1806-1814. Medline

Hromádka T, Zador AM, DeWeese MR (2013) Up states are rare in awake auditory cortex. J Neurophysiol 109:1989-1995. CrossRef Medline

Huang X, Troy WC, Yang Q, Ma H, Laing CR, Schiff SJ, Wu JY (2004) Spiral waves in disinhibited mammalian neocortex. J Neurosci 24:9897-9902. CrossRef Medline

Huang X, Xu W, Liang J, Takagaki K, Gao X, Wu JY (2010) Spiral wave dynamics in neocortex. Neuron 68:978-990. CrossRef Medline

Hussain AKM (1986) Coherent structures and turbulence. J Fluid Mech 173:303-356. CrossRef

Koch C (1999) Biophysics of computation: information processing in single neurons. Oxford: Oxford UP.

Koch KW, Fuster JM (1989) Unit activity in monkey parietal cortex related to haptic perception and temporary memory. Exp Brain Res 76:292-306. Medline

Lefort S, Tomm C, Floyd Sarria JC, Petersen CC (2009) The excitatory neuronal network of the $\mathrm{C} 2$ barrel column in mouse primary somatosensory cortex. Neuron 61:301-316. CrossRef Medline

Levy RB, Reyes AD (2012) Spatial profile of excitatory and inhibitory synaptic connectivity in mouse primary auditory cortex. J Neurosci 32:5609_ 5619. CrossRef Medline

Litwin-Kumar A, Doiron B (2012) Slow dynamics and high variability in balanced cortical networks with clustered connections. Nat Neurosci 15: 1498-1505. CrossRef Medline

Markov NT, Misery P, Falchier A, Lamy C, Vezoli J, Quilodran R, Gariel MA, Giroud P, Ercsey-Ravasz M, Pilaz LJ, Huissoud C, Barone P, Dehay C, Toroczkai Z, Van Essen DC, Kennedy H, Knoblauch K (2011) Weight 
consistency specifies regularities of macaque cortical networks. Cereb Cortex 21:1254-1272. CrossRef Medline

Metzler R, Klafter J (2000) The random walk's guide to anomalous diffusion: a fractional dynamics approach. Phys Rep 339:1-77. CrossRef

Okun M, Lampl I (2008) Instantaneous correlation of excitation and inhibition during ongoing and sensory-evoked activities. Nat Neurosci 11: 535-537. CrossRef Medline

Petersen CC, Hahn TT, Mehta M, Grinvald A, Sakmann B (2003) Interaction of sensory responses with spontaneous depolarization in layer $2 / 3$ barrel cortex. Proc Natl Acad Sci U S A 100:13638-13643. CrossRef Medline

Poulet JF, Petersen CC (2008) Internal brain state regulates membrane potential synchrony in barrel cortex of behaving mice. Nature 454:881-885. CrossRef Medline

Pratt WK (1991) Digital image processing. New York: Wiley.

Press WH, Teukolsky SA, Vetterling WT, Flannery BP (2007) Numerical recipes: the art of scientific computing. Cambridge: Cambridge UP.

Renart A, de la Rocha J, Bartho P, Hollender L, Parga N, Reyes A, Harris KD (2010) The asynchronous state in cortical circuits. Science 327:587-590. CrossRef Medline

Rosenbaum R, Doiron B (2014) Balanced networks of spiking neurons with spatially dependent recurrent connections. Phys Rev X 4:021039.

Rubino D, Robbins KA, Hatsopoulos NG (2006) Propagating waves mediate information transfer in the motor cortex. Nat Neurosci 9:1549-1557. CrossRef Medline

Salinas E, Sejnowski TJ (2000) Impact of correlated synaptic input on output firing rate and variability in simple neuronal models. J Neurosci 20: 6193-6209. Medline

Sato TK, Nauhaus I, Carandini M (2012) Traveling waves in visual cortex. Neuron 75:218-229. CrossRef Medline

Shadlen MN, Newsome WT (1994) Noise, neural codes and cortical organization. Curr Opin Neurobiol 4:569-579. CrossRef Medline

Shadlen MN, Newsome WT (1998) The variable discharge of cortical neurons: implications for connectivity, computation, and information coding. J Neurosci 18:3870-3896. Medline
Smith MA, Kohn A (2008) Spatial and temporal scales of neuronal correlation in primary visual cortex. J Neurosci 28:12591-12603. CrossRef Medline

Softky WR, Koch C (1993) The highly irregular firing of cortical cells is inconsistent with temporal integration of random EPSPs. J Neurosci 13: 334-350. Medline

Stevens CF, Zador AM (1998) Input synchrony and the irregular firing of cortical neurons. Nat Neurosci 1:210-217. CrossRef Medline

Tan AY, Chen Y, Scholl B, Seidemann E, Priebe NJ (2014) Sensory stimulation shifts visual cortex from synchronous to asynchronous states. Nature 509:226-229. CrossRef Medline

Tolhurst DJ, Movshon JA, Dean AF (1983) The statistical reliability of signals in single neurons in cat and monkey visual cortex. Vision Res 23:775785. CrossRef Medline

Vaadia E, Haalman I, Abeles M, Bergman H, Prut Y, Slovin H, Aertsen A (1995) Dynamics of neuronal interactions in monkey cortex in relation to behavioural events. Nature 373:515-518. CrossRef Medline

van Vreeswijk C, Sompolinsky H (1996) Chaos in neuronal networks with balanced excitatory and inhibitory activity. Science 274:1724-1726. CrossRef Medline

van Vreeswijk C, Sompolinsky H (1998) Chaotic balanced state in a model of cortical circuits. Neural Comput 10:1321-1371. CrossRef Medline

Wang XJ (2010) Neurophysiological and computational principles of cortical rhythms in cognition. Physiol Rev 90:1195-1268. CrossRef Medline

Wehr M, Zador AM (2003) Balanced inhibition underlies tuning and sharpens spike timing in auditory cortex. Nature 426:442-446. CrossRef Medline

Wu JY, Xiaoying H, Chuan Z (2008) Propagating waves of activity in the neocortex: what they are, what they do. Neuroscientist 14:487-502. CrossRef Medline

Xue M, Atallah BV, Scanziani M (2014) Equalizing excitation-inhibition ratios across visual cortical neurons. Nature 511:596-600. CrossRef Medline 